\title{
INVERTING A MATRIX FUNCTION AROUND A SINGULARITY VIA LOCAL RANK FACTORIZATION*
}

\author{
MASSIMO FRANCHI ${ }^{\dagger}$ AND PAOLO PARUOLO $\ddagger$
}

\begin{abstract}
This paper proposes a recursive procedure, called the extended local rank factorization (ELRF), that characterizes the order of the pole and the coefficients of the Laurent series representation of the inverse of a regular analytic matrix function around a given point. The ELRF consists in performing a finite sequence of rank factorizations of matrices of nonincreasing dimension, at most equal to the dimension of the original matrix function. Each step of the sequence is associated with a reduced rank condition, while the termination of the ELRF corresponds to a full rank condition; this last step reveals the order of the pole. The Laurent coefficients $B_{n}$ are calculated recursively as $B_{n}=C_{n}+\sum_{k=1}^{n} D_{k} B_{n-k}$, where $C_{n}, D_{k}$ have simple closed form expressions in terms of the quantities generated by the ELRF. It is also shown that the ELRF characterizes the structure of Jordan pairs, Jordan chains, and the local Smith form. The procedure is easily cast in an algorithmic form, and a MATLAB implementation script is provided. It is further found that the ELRF coincides with the complete reduction process (CRP) in Avrachenkov, Haviv, and Howlett [SIAM J. Matrix Anal. Appl., 22 (2001), pp. 1175-1189]. Using this connection, the results on the ELRF provide both an explicit recursive formula for $B_{n}$ implied by the CRP, and the link between the $\mathrm{CRP}$ and the structure of the local Smith form.
\end{abstract}

Key words. matrix valued functions, matrix inversion, analytic perturbation, Laurent series expansion, local Smith form, Jordan pairs

AMS subject classifications. 15A09, 47A05, 47A11, 47A56

DOI. $10.1137 / 140999839$

1. Introduction. Consider a regular analytic matrix function $A(z)$ defined on an open set $U \subseteq \mathbb{C}$, and let

$$
A(z)=\sum_{n=0}^{\infty} A_{n}\left(z-z_{0}\right)^{n}, \quad A_{n} \in \mathbb{C}^{p \times p}, \quad A_{0} \neq 0, \quad z \in U,
$$

be its representation around the point $z_{0} \in U$. Assume that $A\left(z_{0}\right)=A_{0}$ is singular, and let the Laurent representation of the inverse of $A(z)$ be

$$
A(z)^{-1}=\sum_{n=0}^{\infty} B_{n}\left(z-z_{0}\right)^{n-m}, \quad B_{0} \neq 0 .
$$

This paper discusses a recursive procedure to determine the order of the pole of $A(z)^{-1}$ at $z_{0}, m$, and the Laurent coefficients, $\left\{B_{n}\right\}_{n=0}^{\infty}$, given the coefficients of the original matrix function, $\left\{A_{n}\right\}_{n=0}^{\infty}$.

A classical approach to characterizing the relation between (1.1) and (1.2) is via the local spectral theory, based on the concepts of root functions, Jordan chains, and the local Smith form; see $[5,12,13,17]$. The case of matrix polynomials is an

* Received by the editors December 15, 2014; accepted for publication (in revised form) by M. Hochbruck April 14, 2016; published electronically June 21, 2016. This work was supported by the Ministero dell'Istruzione, dell'Università e della Ricerca under MIUR PRIN grant 2010J3LZEN.

http://www.siam.org/journals/simax/37-2/99983.html

$\dagger$ Corresponding author. Sapienza University of Rome, 00185 Rome, Italy (massimo.franchi@ uniroma1.it).

${ }_{\ddagger}^{\ddagger}$ European Commission, Joint Research Centre, I-21027 Ispra (VA), Italy (paolo.paruolo@jrc.ec. europa.eu). 
important special case; see $[14,15,16,28,29,31,32]$ and $[11,30,34,36]$ for matrix polynomials of degree one. The tools derived from the local spectral theory are used in the study of similarity of matrices [11, 30, 34], for the solutions of systems of differential equations $[15,16]$, and in linear control theory $[4,22,27]$, as well as in time series econometrics $[7,8,9,19,26,35]$. The same tools are also employed in numerical algorithms, such as those in [37, 38], for calculating the global Smith form of matrix polynomials and the Laurent representation of the inverse.

Another approach to the calculation of the Laurent coefficients $B_{n}$ was proposed in [3] (see also [23]) and begins by writing the identity $A(z) A(z)^{-1}=I$ as the following linear system in the $A_{n}, B_{n}$ matrices:

$$
\begin{aligned}
A_{0} B_{0} & =0, \\
A_{0} B_{1}+A_{1} B_{0} & =0,
\end{aligned}
$$

In the following, equations in system (1.3) are indexed according to the highest value of the subscript of $B_{n}$; for instance, $A_{0} B_{0}=0$ is referred to as equation 0 . Note that the identity appears in equation $m$, which is the order of the pole.

In [3], the $A_{n}$ matrices (or reduced versions of them) are stacked into appropriate augmented matrices, and the resulting system is solved for the Laurent coefficients $B_{n}$. Because the system (1.3) is singular, its direct solution involves the computation of a (Moore-Penrose) generalized inverse of dimension $m p$. Building on the results of [22] and on the reduction technique developed in [20,21], the authors of [3] further propose a reduction process of system (1.3) based on its singularity. This process can be applied once, giving rise to a one-step reduction process or, recursively, yielding the so-called complete reduction process (CRP).

The CRP is expected to have numerical advantages with respect to the one-step reduction process because it exploits further singularities, although an explicit formula for the resulting Laurent coefficients is not available for the CRP. In addition, the CRP also delivers the order of the pole. If one applies the direct solution or the one-step reduction, the order of the pole must be predetermined, for example using the rank test in [33]. The book [2] contains a thorough treatment of this approach and its extensions; see, e.g., [1] for the case of operator pencils on Banach spaces.

The present paper exploits rank restrictions in (1.3), which are used to rankdecompose some relevant matrices of small dimension (less than or equal to $p$ ), obtaining two new (block-)orthogonal bases. This allows one to define a relevant set of projections of the $B_{n}$ coefficients, for which explicit (recursive) formulae are found; the latter solutions are then rearranged into the general expression

$$
B_{n}=C_{n}+\sum_{k=1}^{n} D_{k} B_{n-k}, \quad n \geq 0
$$

where $C_{n}, D_{k}$ have simple closed form expressions. For a pole of order $m$, there are $m+1$ rank conditions: the first $m$ are reduced rank restrictions, and the last one is 
a full rank condition, and this gives a way to calculate the order of the pole. This procedure is called the extended local rank factorization (ELRF), being an extension of the local rank factorization (LRF) proposed in [10].

The spirit of the ELRF is thus similar to that of Theorem 5 in [22] and also to the rank test in [33], where the order of the pole is established recursively by checking the rank of a sequence of matrices until a full rank condition is satisfied. The main difference is that instead of working on stacked system matrices whose dimension increases with $m$, the present approach works on matrices whose dimension is at most equal to the dimension of the original matrix function, $p$, and that decreases with $m$.

The sequence of rank factorizations is further shown to deliver the partial multiplicities and the number of partial multiplicities of a given value, i.e., the local Smith form, and to provide a construction of an extended canonical system of root functions of $A(z)$ at $z_{0}$. In this way, the structure of a Jordan pair is fully characterized and full information on Jordan chains is available. These theoretical results are translated into an algorithmic form, and a MATLAB script that implements the ELRF is provided in the supplementary material (99983_01.pdf [local/web 162KB]), which is linked from the main article webpage.

The connections with the CRP in [3] are discussed, and it is shown that the CRP coincides with the ELRF. Via this equivalence, the characteristics of the CRP are linked to the structure of the local Smith form and it is shown that the number of reductions in the CRP is equal to the number of distinct nonzero partial multiplicities and each reduction step decreases the dimension of the coefficients by the number of partial multiplicities that are equal to a given value.

The rest of the paper is organized as follows. Section 2 motivates the techniques used in the paper via an illustration on poles of orders one and two; section 3 contains the general formulation and results; section 4 discusses the connections with the local Smith form, the extended canonical system of root functions, Jordan chains, and Jordan pairs; section 5 presents the algorithmic implementation of the ELRF and its relation to the CRP in [3]; section 6 discusses the computational complexity of the algorithm; section 7 illustrates the results via a numerical example; and section 8 concludes. The supplementary material contains the MATLAB script that implements the ELRF.

2. Motivation. This section motivates and illustrates the technique presented in the paper by discussing the cases of poles of orders one and two; the general formulation is presented in section 3. In particular, the cases considered in this section make it clear how the sequence of rank factorizations can be used to define a new block-orthogonal basis, which is then employed to decompose $B_{n}$ into relevant projections.

The techniques presented in the paper make repeated use of rank factorizations and projections, whose notation is introduced here: given a $p \times p$ matrix $\varphi$ of rank $0<r<p$, its rank factorization is written as $\varphi=-\alpha \beta^{\prime}$, where $\alpha$ and $\beta$ are $p \times r$ full column rank matrices that span the column space $(\operatorname{col} \varphi)$ and the row space $\left(\operatorname{col} \varphi^{\prime}\right)$ of $\varphi$, respectively; the negative sign is chosen for convenience in the calculations. The matrix $\varphi_{\perp}$ denotes a $p \times(p-r)$ full column rank matrix that $\operatorname{spans} \operatorname{col}^{\perp} \varphi$, the orthogonal complement of $\operatorname{col} \varphi=\operatorname{col} \alpha$. The orthogonal projection matrix onto $\operatorname{col} \varphi$ is denoted by $P_{\alpha}:=\bar{\alpha} \alpha^{\prime}=\alpha \bar{\alpha}^{\prime}$, where $\bar{\alpha}:=\alpha\left(\alpha^{\prime} \alpha\right)^{-1}$, and it has rank $r$; $P_{\alpha_{\perp}}:=I-P_{\alpha}=\bar{\alpha}_{\perp} \alpha_{\perp}^{\prime}=\alpha_{\perp} \bar{\alpha}_{\perp}^{\prime}$ of rank $p-r$ is the orthogonal projection matrix onto $\operatorname{col}^{\perp} \varphi$. Similarly, for $\operatorname{col} \varphi^{\prime}$, one can define $P_{\beta}$ and $P_{\beta_{\perp}}$. When $r=0$, i.e., $\varphi=0$, one lets $\alpha=\beta=\bar{\alpha}=\bar{\beta}=0$ and $\alpha_{\perp}=\beta_{\perp}=\bar{\alpha}_{\perp}=\bar{\beta}_{\perp}=I$. When $r=p$, i.e., $\varphi$ is of 
full rank, one can take either $\alpha$ or $\beta$ equal to $I$ and let $\alpha_{\perp}=\beta_{\perp}=\bar{\alpha}_{\perp}=\bar{\beta}_{\perp}=0$.

Before considering the special cases of poles of orders $m=1,2$, some general observations are presented. Recall that the equations in (1.3) are referred to according to the appropriate order index $0,1, \ldots$ Consider equation 0 in (1.3) and observe that, because $A_{0} \neq 0$ is singular, it can be rank-decomposed as $A_{0}=-\alpha_{0} \beta_{0}^{\prime}$, where $\alpha_{0}$ and $\beta_{0}$ have rank $0<r_{0}:=\operatorname{rank} A_{0}<p$. This implies that the associated projection matrices $P_{\alpha_{0}}, P_{\alpha_{0 \perp}}$ and $P_{\beta_{0}}, P_{\beta_{0 \perp}}$ are all different from 0 . Note that equation 0 implies $\beta_{0}^{\prime} B_{0}=0$ so that

$$
B_{0}=P_{\beta_{0}} B_{0}+P_{\beta_{0 \perp}} B_{0}=P_{\beta_{0 \perp}} B_{0}
$$

can be substituted in (1.3).

Next, replacing $A_{0}=-\alpha_{0} \beta_{0}^{\prime}$ in a generic equation $n$ in (1.3) and using Kronecker's delta $\delta_{n, m}$ (defined as $\delta_{m, m}:=1$ and $\delta_{n, m}:=0$ otherwise), one can rewrite equation $n$ as

$$
\alpha_{0} \beta_{0}^{\prime} B_{n}=\sum_{k=1}^{n} A_{k} B_{n-k}-\delta_{n, m} I .
$$

Note that (2.2) implies $\beta_{0}^{\prime} B_{n}=\bar{\alpha}_{0}^{\prime} \sum_{k=1}^{n} A_{k} B_{n-k}-\delta_{n, m} \bar{\alpha}_{0}^{\prime}$ and hence

$$
P_{\beta_{0}} B_{n}=\bar{\beta}_{0} \bar{\alpha}_{0}^{\prime} \sum_{k=1}^{n} A_{k} B_{n-k}-\delta_{n, m} \bar{\beta}_{0} \bar{\alpha}_{0}^{\prime} .
$$

This shows that equation $n$ gives information on $P_{\beta_{0}} B_{n}$ but not on the complementary part $P_{\beta_{0 \perp}} B_{n}$. Because $B_{n}$ does not appear in equations 0 to $n-1$, this information must necessarily come from the subsequent equations. The next two subsections show what happens in the cases $m=1$ or 2 .

2.1. Pole of order 1. In case $m=1$, system (1.3) reads as

$$
\begin{aligned}
-\alpha_{0} \beta_{0}^{\prime} B_{0} & =0, \\
-\alpha_{0} \beta_{0}^{\prime} B_{1}+A_{1} P_{\beta_{0 \perp}} B_{0} & =I, \\
-\alpha_{0} \beta_{0}^{\prime} B_{2}+A_{1} B_{1}+A_{2} P_{\beta_{0 \perp}} B_{0} & =0,
\end{aligned}
$$

Here it is shown that all the information on $P_{\beta_{0 \perp}} B_{0}$ is found in equation 1. In fact, premultiply equation 1 by $P_{\alpha_{0 \perp}}$ to find that

$$
\left(P_{\alpha_{0 \perp}} A_{1} P_{\beta_{0 \perp}}\right) B_{0}=P_{\alpha_{0 \perp}} .
$$

Because rank $P_{\alpha_{0 \perp}}=\operatorname{rank} P_{\beta_{0 \perp}}=p-r_{0}$, one has $\operatorname{rank}\left(P_{\alpha_{0 \perp}} A_{1} P_{\beta_{0 \perp}}\right) \leq p-r_{0}$, and hence (2.4) is consistent if and only if $P_{\alpha_{0 \perp}} A_{1} P_{\beta_{0 \perp}}$ has maximal rank $p-r_{0}$. Because $P_{\alpha_{0 \perp}} A_{1} P_{\beta_{0 \perp}}=\bar{\alpha}_{0 \perp} \alpha_{0 \perp}^{\prime} A_{1} \beta_{0 \perp} \bar{\beta}_{0 \perp}^{\prime}$, the maximal rank condition $\operatorname{rank}\left(P_{\alpha_{0 \perp}} A_{1} P_{\beta_{0 \perp}}\right)=$ $p-r_{0}$ is equivalent to the full rank condition

$$
r_{1}=r_{1}^{\max }, \quad \text { where } \quad r_{1}:=\operatorname{rank} \alpha_{0 \perp}^{\prime} A_{1} \beta_{0 \perp} \quad \text { and } \quad r_{1}^{\max }:=p-r_{0},
$$

and $\alpha_{0 \perp}^{\prime} A_{1} \beta_{0 \perp}$ is a square matrix of dimension $r_{1}^{\max }$. This corresponds to the condition in Theorem 3 of [22] and to Theorem 4.1 in [24]. 
Note that in this case the full rank condition (2.5) implies that the rank factorization $\alpha_{0 \perp}^{\prime} A_{1} \beta_{0 \perp}=-\xi_{1} \eta_{1}^{\prime}$ is such that $\xi_{1}$ and $\eta_{1}$ are square nonsingular matrices of dimension $r_{1}^{\max }$; note also that, by premultiplying $\alpha_{0 \perp}^{\prime} A_{1} \beta_{0 \perp}=-\xi_{1} \eta_{1}^{\prime}$ by $\bar{\alpha}_{0 \perp}$ and postmultiplying it by $\bar{\beta}_{0 \perp}^{\prime}$, the rank factorization can be written as

$$
P_{\alpha_{0 \perp}} A_{1} P_{\beta_{0 \perp}}=-\alpha_{1} \beta_{1}^{\prime}, \quad \text { where } \quad \alpha_{1}:=\bar{\alpha}_{0 \perp} \xi_{1} \quad \text { and } \quad \beta_{1}:=\bar{\beta}_{0 \perp} \eta_{1} .
$$

Thanks to the full rank condition (2.5), one then has

$$
\operatorname{col}\left(\alpha_{0}, \alpha_{1}\right)=\operatorname{col}\left(\beta_{0}, \beta_{1}\right)=\mathbb{C}^{p}
$$

because (i) $\alpha_{0}$ and $\alpha_{1}$ are orthogonal by construction, (ii) each of them has full column rank, and (iii) the sum of their number of columns $r_{0}+r_{1}$ equals $p$. A similar argument holds for $\beta_{0}, \beta_{1}$. This implies

$$
P_{\alpha_{1}}=P_{\alpha_{0 \perp}}, \quad P_{\beta_{1}}=P_{\beta_{0 \perp}}, \quad \text { and hence } \quad I=P_{\alpha_{0}}+P_{\alpha_{1}}=P_{\beta_{0}}+P_{\beta_{1}} .
$$

Using (2.6), one can rewrite (2.4) as $-\alpha_{1} \beta_{1}^{\prime} B_{0}=P_{\alpha_{1}}$, which implies $\beta_{1}^{\prime} B_{0}=-\bar{\alpha}_{1}^{\prime}$ and $P_{\beta_{1}} B_{0}=-\bar{\beta}_{1} \bar{\alpha}_{1}^{\prime}$; hence

$$
B_{0}=P_{\beta_{1}} B_{0}=-\bar{\beta}_{1} \bar{\alpha}_{1}^{\prime} .
$$

Summing up, when $m=1$, equation 1 contains all the information on $P_{\beta_{0 \perp}} B_{0}$, and thus one can solve for $B_{0}$ using equations 0 and 1 only. This is in line with Theorem 1 in [3], which states that $m+1$ equations are needed to calculate $B_{n}$ given knowledge of $B_{0}, \ldots, B_{n-1}$.

Similarly, it can be shown that the information on the second Laurent coefficient $B_{1}$ comes from equations 1 and 2. In fact, consider $B_{1}$ and decompose it into its projections on $\operatorname{col} \beta_{0}$ and $\operatorname{col} \beta_{1}$, i.e., $B_{1}=P_{\beta_{0}} B_{1}+P_{\beta_{1}} B_{1}$. From equation 1 one finds that $\beta_{0}^{\prime} B_{1}=\bar{\alpha}_{0}^{\prime}\left(A_{1} B_{0}-I\right)$, and hence one has

$$
P_{\beta_{0}} B_{1}=\bar{\beta}_{0} \bar{\alpha}_{0}^{\prime}\left(A_{1} B_{0}-I\right) .
$$

Information on $P_{\beta_{1}} B_{1}$ is obtained by premultiplying equation 2 by $P_{\alpha_{1}}$ to find that $-P_{\alpha_{1}} A_{1} B_{1}=P_{\alpha_{1}} A_{2} B_{0}$. Inserting $I=P_{\beta_{0}}+P_{\beta_{1}}$ between $A_{1}$ and $B_{1}$ and using (2.6), one has $\alpha_{1} \beta_{1}^{\prime} B_{1}=P_{\alpha_{1}}\left(A_{2} B_{0}+A_{1} P_{\beta_{0}} B_{1}\right)$. Substituting $P_{\beta_{0}} B_{1}=\bar{\beta}_{0} \bar{\alpha}_{0}^{\prime}\left(A_{1} B_{0}-I\right)$ from (2.8) in the last expression and defining $A_{2,1}:=A_{2}+A_{1} \bar{\beta}_{0} \bar{\alpha}_{0}^{\prime} A_{1}$, one then finds that

$$
\alpha_{1} \beta_{1}^{\prime} B_{1}=P_{\alpha_{1}} A_{2,1} B_{0}-P_{\alpha_{1}} A_{1} \bar{\beta}_{0} \bar{\alpha}_{0}^{\prime} .
$$

This implies $\beta_{1}^{\prime} B_{1}=\bar{\alpha}_{1}^{\prime}\left(A_{2,1} B_{0}-A_{1} \bar{\beta}_{0} \bar{\alpha}_{0}^{\prime}\right)$ and hence

$$
P_{\beta_{1}} B_{1}=\bar{\beta}_{1} \bar{\alpha}_{1}^{\prime}\left(A_{2,1} B_{0}-A_{1} \bar{\beta}_{0} \bar{\alpha}_{0}^{\prime}\right) .
$$

Substituting (2.8) and (2.10) in $B_{1}=P_{\beta_{0}} B_{1}+P_{\beta_{1}} B_{1}$, one finds that

$$
B_{1}=C_{1}+D_{1} B_{0},
$$

where $C_{1}:=-\bar{\beta}_{0} \bar{\alpha}_{0}^{\prime}-\bar{\beta}_{1} \bar{\alpha}_{1}^{\prime} A_{1} \bar{\beta}_{0} \bar{\alpha}_{0}^{\prime}$ and $D_{1}:=\bar{\beta}_{0} \bar{\alpha}_{0}^{\prime} A_{1}+\bar{\beta}_{1} \bar{\alpha}_{1}^{\prime} A_{2,1}$. This shows that when $m=1$ one can solve for $B_{1}$ using equations 1 and 2 and the previous expression for $B_{0}$. Similarly, for each $n$ one can write $B_{n}=P_{\beta_{0}} B_{n}+P_{\beta_{1}} B_{n}$, and solve equation $n$ for $P_{\beta_{0}} B_{n}$ and equation $n+1$ for $P_{\beta_{1}} B_{n}$, using knowledge of $B_{0}, \ldots, B_{n-1}$. 
The above illustrates the role of the "full rank condition", equivalently stated as the maximal rank of $P_{\alpha_{0 \perp}} A_{1} P_{\beta_{0 \perp}}$ or the full rank of $\alpha_{0 \perp}^{\prime} A_{1} \beta_{0 \perp}$ (i.e., $r_{1}=r_{1}^{\max }$ ), or as $\operatorname{col}\left(\alpha_{0}, \alpha_{1}\right)=\operatorname{col}\left(\beta_{0}, \beta_{1}\right)=\mathbb{C}^{p}$, where the latter and block-orthogonality of the bases imply the projection identities $I=P_{\alpha_{0}}+P_{\alpha_{1}}=P_{\beta_{0}}+P_{\beta_{1}}$; see (2.4), (2.5), and (2.7). Note that this full rank condition applies to equation 1 due to the presence of $I$ on the right-hand side (RHS), corresponding to the order of the pole $m=1$. More generally, for a pole of order $m$, the full rank condition applies to equation $m$, and this gives a way of finding the order of the pole by checking the full rank condition, as shown in section 3 .

2.2. Pole of order 2. In case $m=2$, system (1.3) reads as

$$
\begin{aligned}
-\alpha_{0} \beta_{0}^{\prime} B_{0} & =0, \\
-\alpha_{0} \beta_{0}^{\prime} B_{1}+A_{1} P_{\beta_{0 \perp}} B_{0} & =0, \\
-\alpha_{0} \beta_{0}^{\prime} B_{2}+A_{1} B_{1}+A_{2} P_{\beta_{0 \perp}} B_{0} & =I, \\
-\alpha_{0} \beta_{0}^{\prime} B_{3}+A_{1} B_{2}+A_{2} B_{1}+A_{3} P_{\beta_{0 \perp}} B_{0} & =0,
\end{aligned}
$$

Proceeding as above in equation 1, one finds that

$$
\left(P_{\alpha_{0 \perp}} A_{1} P_{\beta_{0 \perp}}\right) B_{0}=0
$$

i.e., using the rank factorization (2.6), $-\alpha_{1} \beta_{1}^{\prime} B_{0}=0$, which implies $\beta_{1}^{\prime} B_{0}=0$ and $P_{\beta_{1}} B_{0}=0$. If $r_{1}=r_{1}^{\max }$, one would have (2.7) and hence $B_{0}=0$, which in the present case $m=2$ would give a contradiction. This means that when $m>1$ the following reduced rank condition must hold:

$$
r_{1}<r_{1}^{\max }, \quad \text { where } r_{1}:=\operatorname{rank} \alpha_{0 \perp}^{\prime} A_{1} \beta_{0 \perp} \quad \text { and } \quad r_{1}^{\max }:=p-r_{0},
$$

and in this case the rank factorization $\alpha_{0 \perp}^{\prime} A_{1} \beta_{0 \perp}=-\xi_{1} \eta_{1}^{\prime}$, or equivalently (2.6), involves a reduction of rank. Note that here $\xi_{1}, \eta_{1}$ are $r_{1}^{\max } \times r_{1}$ matrices and $\operatorname{col} \alpha_{1}$, $\operatorname{col} \beta_{1}$ are $r_{1}$-dimensional subspaces of $\operatorname{col} \alpha_{0 \perp}, \operatorname{col} \beta_{0 \perp}$, respectively.

Letting $a_{2}:=\left(\alpha_{0}, \alpha_{1}\right)$ and $b_{2}:=\left(\beta_{0}, \beta_{1}\right)$, one has $\operatorname{col} \alpha_{0 \perp}=\operatorname{col}\left(\alpha_{1}, a_{2 \perp}\right)$ and $\operatorname{col} \beta_{0 \perp}=\operatorname{col}\left(\beta_{1}, b_{2 \perp}\right)$, where $a_{2 \perp}$ (respectively, $\left.b_{2 \perp}\right)$ spans the $\left(p-r_{0}-r_{1}\right)$-dimensional complementary subspace of $\operatorname{col} \alpha_{0 \perp}$ (respectively, $\operatorname{col} \beta_{0 \perp}$ ) not generated by $\alpha_{1}$ (respectively, $\beta_{1}$ ). Hence in this case one needs to decompose the Laurent coefficients in more than two projections; in particular, note that using $I=P_{\alpha_{0}}+P_{\alpha_{1}}+P_{a_{2 \perp}}=$ $P_{\beta_{0}}+P_{\beta_{1}}+P_{b_{2 \perp}}$, one has $B_{n}=P_{\beta_{0}} B_{n}+P_{\beta_{1}} B_{n}+P_{b_{2 \perp}} B_{n}$.

For $n=0$, one finds that $B_{0}=P_{b_{2} \perp} B_{0}$. In fact, $P_{\beta_{0}} B_{0}=0$ holds from equation 0 and $P_{\beta_{1}} B_{0}=0$ holds from equation 1 because $\alpha_{1} \beta_{1}^{\prime} B_{0}=0$ (see (2.12)) implies $P_{\beta_{1}} B_{0}=0$. Information on $P_{b_{2} \perp} B_{0}$ is found by premultiplying equation 2 by $P_{\alpha_{0 \perp}}$ to find that $P_{\alpha_{0 \perp}} A_{1} B_{1}+P_{\alpha_{0 \perp}} A_{2} P_{b_{2} \perp} B_{0}=P_{\alpha_{0 \perp}}$; inserting $I=P_{\beta_{0}}+P_{\beta_{0 \perp}}$ between $A_{1}$ and $B_{1}$ and using (2.6), one then finds that

$$
-\alpha_{1} \beta_{1}^{\prime} B_{1}+P_{\alpha_{0 \perp}} A_{1} P_{\beta_{0}} B_{1}+P_{\alpha_{0 \perp}} A_{2} P_{b_{2} \perp} B_{0}=P_{\alpha_{0 \perp}} .
$$

Observe that equation 1 implies $\beta_{0}^{\prime} B_{1}=\bar{\alpha}_{0}^{\prime} A_{1} P_{b_{2} \perp} B_{0}$, i.e., $P_{\beta_{0}} B_{1}=\bar{\beta}_{0} \bar{\alpha}_{0}^{\prime} A_{1} P_{b_{2} \perp} B_{0}$; hence premultiplying (2.14) by $P_{a_{2 \perp}}$ and rearranging, one finds that

$$
\left(P_{a_{2} \perp} A_{2,1} P_{b_{2} \perp}\right) B_{0}=P_{a_{2 \perp}}, \quad A_{2,1}:=A_{2}+A_{1} \bar{\beta}_{0} \bar{\alpha}_{0}^{\prime} A_{1} .
$$


This equation has the same format of (2.4), and the same reasoning applies: because $\operatorname{rank} P_{a_{2 \perp}}=\operatorname{rank} P_{b_{2 \perp}}=p-r_{0}-r_{1}$, one has $\operatorname{rank}\left(P_{a_{2 \perp}} A_{2,1} P_{b_{2} \perp}\right) \leq p-r_{0}-r_{1}$, and hence (2.15) is consistent if and only if $P_{a_{2} \perp} A_{2,1} P_{b_{2 \perp}}$ has maximal rank $p-$ $r_{0}-r_{1}$. Because $P_{a_{2 \perp}} A_{2,1} P_{b_{2} \perp}=\bar{a}_{2 \perp}\left(a_{2 \perp}^{\prime} A_{2,1} b_{2 \perp}\right) \bar{b}_{2 \perp}^{\prime}$, the maximal rank condition $\operatorname{rank}\left(P_{a_{2} \perp} A_{2,1} P_{b_{2} \perp}\right)=p-r_{0}-r_{1}$ is equivalent to the full rank condition

$$
r_{2}=r_{2}^{\max }, \quad \text { where } r_{2}:=\operatorname{rank} a_{2 \perp}^{\prime} A_{2,1} b_{2 \perp} \quad \text { and } \quad r_{2}^{\max }:=p-r_{0}-r_{1},
$$

and $a_{2 \perp}^{\prime} A_{2,1} b_{2 \perp}$ is a square matrix of dimension $r_{2}^{\max }$. This corresponds to Theorem 3 in $[25]$.

Note that in this case the full rank condition (2.16) implies that the rank factorization $a_{2 \perp}^{\prime} A_{2,1} b_{2 \perp}=-\xi_{2} \eta_{2}^{\prime}$ is such that $\xi_{2}$ and $\eta_{2}$ are square nonsingular matrices of dimension $r_{2}^{\max }$; observe also that, by premultiplying $a_{2 \perp}^{\prime} A_{2,1} b_{2 \perp}=-\xi_{2} \eta_{2}^{\prime}$ by $\bar{a}_{2 \perp}$ and postmultiplying it by $\bar{b}_{2 \perp}^{\prime}$, the rank factorization can be written as

$$
P_{a_{2} \perp} A_{2,1} P_{b_{2} \perp}=-\alpha_{2} \beta_{2}^{\prime}, \quad \text { where } \quad \alpha_{2}:=\bar{a}_{2 \perp} \xi_{2} \quad \text { and } \quad \beta_{2}:=\bar{b}_{2 \perp} \eta_{2} .
$$

Thanks to the full rank condition (2.16), one then has

$$
\operatorname{col}\left(\alpha_{0}, \alpha_{1}, \alpha_{2}\right)=\operatorname{col}\left(\beta_{0}, \beta_{1}, \beta_{2}\right)=\mathbb{C}^{p}
$$

because (i) $\alpha_{0}, \alpha_{1}, \alpha_{2}$ are orthogonal by construction, (ii) each of them has full column rank, and (iii) the sum of their number of columns $r_{0}+r_{1}+r_{2}$ equals $p$. A similar argument holds for $\beta_{0}, \beta_{1}, \beta_{2}{ }^{1}$ This implies

$$
P_{\alpha_{2}}=P_{a_{2 \perp}}, \quad P_{\beta_{2}}=P_{b_{2 \perp}}, \quad \text { and hence } \quad I=P_{\alpha_{0}}+P_{\alpha_{1}}+P_{\alpha_{2}}=P_{\beta_{0}}+P_{\beta_{1}}+P_{\beta_{2}} .
$$

Using $P_{\alpha_{2}} A_{2,1} P_{\beta_{2}}=-\alpha_{2} \beta_{2}^{\prime}$, one can then rewrite (2.15) as $-\alpha_{2} \beta_{2}^{\prime} B_{0}=P_{\alpha_{2}}$, which implies $\beta_{2}^{\prime} B_{0}=-\bar{\alpha}_{2}^{\prime}$ and $P_{\beta_{2}} B_{0}=-\bar{\beta}_{2} \bar{\alpha}_{2}^{\prime}$. Hence

$$
B_{0}=P_{\beta_{2}} B_{0}=-\bar{\beta}_{2} \bar{\alpha}_{2}^{\prime} .
$$

Summing up, when $m=2$ there are three rank conditions: (i) $A_{0}=-\alpha_{0} \beta_{0}^{\prime}$, the first reduced rank condition; (ii) (2.13), the second reduced rank condition; (iii) the full rank condition (2.16). The meaning of this sequence of rank conditions can be described as follows: (i) establishes that $m>0$; (ii) establishes that $m>1$; (iii) establishes that $m=2$.

As shown in section 3 below, this is true in general: for a pole of order $m$ there are $m+1$ rank conditions; the first $j=1, \ldots, m$ are reduced rank conditions that establish that the order of the pole is greater than $j-1$, and the last one is the full rank condition that proves that the order of the pole is exactly $m$. These rank factorizations deliver two new bases of $\mathbb{C}^{p}$, namely $\left(\alpha_{0}, \ldots, \alpha_{m}\right)$ and $\left(\beta_{0}, \ldots, \beta_{m}\right)$, with mutually orthogonal components; this gives rise to the projection identities $I=\sum_{j=0}^{m} P_{\alpha_{j}}=\sum_{j=0}^{m} P_{\beta_{j}}$. Each Laurent coefficient $B_{n}$ can then be decomposed into the sum of $m+1$ components $B_{n}=P_{\beta_{0}} B_{n}+\cdots+P_{\beta_{m}} B_{n}$. Equation $j+n$ of (1.3) is solved for component $P_{\beta_{j}} B_{n}$ in terms of $B_{n-1}, \ldots, B_{0}$. The results are then rearranged and expressed recursively as $B_{n}=C_{n}+\sum_{k=1}^{n} D_{k} B_{n-k}$, where $C_{n}, D_{k}$ have simple closed form expressions in terms of the matrices defined by the rank factorizations. This is the structure of the results at the heart of the ELRF presented in the following sections.

\footnotetext{
${ }^{1}$ Indeed, $\alpha_{1}$ and $\beta_{1}$ can be 0 ; see Remark 3.2 below.
} 
3. Laurent coefficients. This section contains the main results. Lemma 3.1 presents a rewriting of a generic equation in system (1.3) that is at the basis of the ELRF; Theorem 3.3 provides the expression for a projection of a Laurent coefficient in terms of the previous Laurent coefficients; Theorem 3.4 relates the rank conditions in system (1.3) with the order of the pole of the inverse; finally, Theorem 3.5 provides the recursive formula for the Laurent coefficients.

Results are based on the following lemma, which is proved by manipulating the identity $A(z) A(z)^{-1}=I$ written in system form as in (1.3). Recall that $P_{\varphi}$ indicates the orthogonal projection matrix onto $\operatorname{col} \varphi$ and $P_{\varphi_{\perp}}$ denotes the orthogonal projection matrix onto $\operatorname{col}^{\perp} \varphi$; further, let $\bar{\varphi}:=\varphi\left(\varphi^{\prime} \varphi\right)^{-1}$ and set $\bar{\varphi}=0$ when $\varphi=0$.

Recall that in this paper it is assumed that $A\left(z_{0}\right)=A_{0} \neq 0$ is singular, say of rank $0<r_{0}<p$. The following lemma defines a sequence of rank factorizations that is later shown to be relevant for the inversion of $A(z)$ at $z=z_{0}$.

Lemma 3.1 (Extended local rank factorization of system (1.3)). Consider the rank factorization $A_{0}=-\alpha_{0} \beta_{0}^{\prime}$ and let $\alpha_{j}, \beta_{j}, j=1,2, \ldots$, be defined by the rank factorization

$$
P_{a_{j \perp}} A_{j, 1} P_{b_{j \perp}}=-\alpha_{j} \beta_{j}^{\prime}, \quad a_{j}:=\left(\alpha_{0}, \ldots, \alpha_{j-1}\right), \quad b_{j}:=\left(\beta_{0}, \ldots, \beta_{j-1}\right),
$$

where $A_{s, k}$ is defined by the recursions

$$
A_{s, k}:=\left\{\begin{array}{cc}
A_{k} & \text { for } s=1, \\
A_{s-1, k+1}+A_{s-1,1} \sum_{i=0}^{s-2} \bar{\beta}_{i} \bar{\alpha}_{i}^{\prime} A_{i+1, k} & \text { otherwise. }
\end{array}\right.
$$

Then equation $n \geq j=0,1, \ldots$ in system (1.3) can be written as

$$
\alpha_{j} \beta_{j}^{\prime} B_{n-j}=P_{a_{j \perp}} \sum_{k=1}^{n-j} A_{j+1, k} B_{n-j-k}+P_{a_{j \perp}} C_{j+1, n-j},
$$

where $C_{s, k}$ is defined by the recursions

$$
C_{s, k}:=\left\{\begin{array}{cc}
-\delta_{k, m} I & \text { for } s=1, \\
C_{s-1, k+1}+A_{s-1,1} \sum_{i=0}^{s-2} \bar{\beta}_{i} \bar{\alpha}_{i}^{\prime} C_{i+1, k} & \text { otherwise. }
\end{array}\right.
$$

Proof. The proof is reached by induction. For $j=0,(3.3)$ reads as $\alpha_{0} \beta_{0}^{\prime} B_{n}=$ $P_{a_{0 \perp}} \sum_{k=1}^{n} A_{1, k} B_{n-k}+P_{a_{0 \perp}} C_{1, n}$; by definition, $A_{1, k}=A_{k}, C_{1, n}=-\delta_{n, m} I$, and $a_{0}=b_{0}=0$, which implies $P_{a_{0 \perp}}=P_{b_{0 \perp}}=I$. Hence (3.3) for $j=0$ coincides with (2.2). Next assume that (3.3) holds for $j=0, \ldots, \ell-1$ for some $\ell>1$; one wishes to show that it also holds for $j=\ell$. Write (3.3) for $j=\ell-1, \alpha_{\ell-1} \beta_{\ell-1}^{\prime} B_{n-\ell+1}=$ $P_{a_{\ell-1 \perp}} \sum_{k=1}^{n-\ell+1} A_{\ell, k} B_{n-\ell+1-k}+P_{a_{\ell-1 \perp}} C_{\ell, n-\ell+1}$, premultiply by $P_{a_{\ell \perp}}$ and rearrange terms to find that

$$
0=P_{a_{\ell \perp}} A_{\ell, 1} B_{n-\ell}+P_{a_{\ell \perp}} \sum_{k=1}^{n-\ell} A_{\ell, k+1} B_{n-\ell-k}+P_{a_{\ell \perp}} C_{\ell, n-\ell+1}:=U+V,
$$

where $a_{\ell}:=\left(\alpha_{0}, \ldots, \alpha_{\ell-1}\right), U:=P_{a_{\ell \perp}} A_{\ell, 1} B_{n-\ell}$, and $V$ is defined consequently. Next, let $b_{\ell}:=\left(\beta_{0}, \ldots, \beta_{\ell-1}\right)$ and use projections, inserting $I=P_{b_{\ell}}+P_{b_{\ell \perp}}$ between $A_{\ell, 1}$ and $B_{n-\ell}$ in $U$; one finds that

$$
U=P_{a_{\ell \perp}} A_{\ell, 1} P_{b_{\ell \perp}} B_{n-\ell}+P_{a_{\ell \perp}} A_{\ell, 1} P_{b_{\ell}} B_{n-\ell}=: U_{1}+U_{2} .
$$


Substituting $P_{b_{\ell}}=P_{\beta_{0}}+\cdots+P_{\beta_{\ell-1}}$, one has $U_{2}=P_{a_{\ell \perp}} A_{\ell, 1} \sum_{i=0}^{\ell-1} P_{\beta_{i}} B_{n-\ell}$; by the induction assumption,

$$
P_{\beta_{i}} B_{n-\ell}=\bar{\beta}_{i} \bar{\alpha}_{i}^{\prime} \sum_{k=1}^{n-\ell} A_{i+1, k} B_{n-\ell-k}+\bar{\beta}_{i} \bar{\alpha}_{i}^{\prime} C_{i+1, n-\ell}
$$

which is derived from (3.3) replacing $n$ with $n-\ell+j$ and $j$ with $i$. Substituting in $U_{2}$, one finds that

$$
U_{2}=P_{a_{\ell \perp}} \sum_{k=1}^{n-\ell}\left(A_{\ell, 1} \sum_{i=0}^{\ell-1} \bar{\beta}_{i} \bar{\alpha}_{i}^{\prime} A_{i+1, k}\right) B_{n-\ell-k}+P_{a_{\ell \perp}} A_{\ell, 1} \sum_{i=0}^{\ell-1} \bar{\beta}_{i} \bar{\alpha}_{i}^{\prime} C_{i+1, n-\ell} ;
$$

hence using (3.2) and (3.4), one has $U_{2}+V=P_{a_{\ell \perp}} \sum_{k=1}^{n-\ell} A_{\ell+1, k} B_{n-\ell-k}+P_{a_{\ell \perp}} C_{\ell+1, n-\ell}$ so that substituting the rank factorization $P_{a_{\ell \perp}} A_{\ell, 1} P_{b_{\ell \perp}}=-\alpha_{\ell} \beta_{\ell}^{\prime}$ in $U_{1}$ and rearranging terms, (3.5) is rewritten as

$$
\alpha_{\ell} \beta_{\ell}^{\prime} B_{n-\ell}=P_{a_{\ell \perp}} \sum_{k=1}^{n-\ell} A_{\ell+1, k} B_{n-\ell-k}+P_{a_{\ell \perp}} C_{\ell+1, n-\ell} .
$$

This shows that (3.3) holds for $j=\ell$.

Remark $3.2\left(\alpha_{j}\right.$ and $\left.\beta_{j}\right)$. Because rank $P_{a_{j \perp}} A_{j, 1} P_{b_{j \perp}}=\operatorname{rank} a_{j \perp}^{\prime} A_{j, 1} b_{j \perp}$, one can replace (3.1) with the rank factorization

$$
\begin{gathered}
a_{j \perp}^{\prime} A_{j, 1} b_{j \perp}=-\xi_{j} \eta_{j}^{\prime}, \quad a_{j}:=\left(\alpha_{0}, \ldots, \alpha_{j-1}\right), \\
\alpha_{j}:=\bar{a}_{j \perp} \xi_{j}, \quad b_{j}:=\left(\beta_{0}, \ldots, \beta_{j-1}\right), \quad \beta_{j}:=\bar{b}_{j \perp} \eta_{j} .
\end{gathered}
$$

This shows that the rank factorizations can be performed on matrices of nonincreasing dimensions, all less than or equal to $p$. In the following, let $r_{j}:=\operatorname{rank} a_{j \perp}^{\prime} A_{j, 1} b_{j \perp}$ indicate the rank of the $j$ th rank factorization and $r_{j}^{\max }:=p-\sum_{i=0}^{j-1} r_{i}$ its maximal value.

It is possible that $\alpha_{j}, \beta_{j}, j=1,2, \ldots$, in (3.1) have rank $r_{j}$ equal to 0 . In this case, both sides of (3.1) are equal to 0 . Note also that, as $j$ increases, $\operatorname{col} a_{j}$ and $\operatorname{col} b_{j}$ are nondecreasing and eventually coincide with $\mathbb{C}^{p}$ for some $j=s$; for subsequent values of $j, j>s$, the left-hand side (LHS) of (3.1) is equal to 0 , because the relevant orthogonal complements have dimension 0 , and hence all subsequent $\alpha_{j}, \beta_{j}$ are equal to 0 .

Finally, observe that $\beta_{s}$ is by construction orthogonal to $\beta_{j}$ for $j=0, \ldots, s-1$, i.e., $\beta_{s}^{\prime} \beta_{j}=0$; as a result, all $\beta_{j}$ 's are mutually orthogonal. A similar block-orthogonality holds for $\alpha_{j}$ s. This fact is indicated in the following saying that $\left\{\alpha_{j}\right\}$ and $\left\{\beta_{j}\right\}$ are block-orthogonal sets of matrices.

Replacing $n-j$ with $n$ in (3.3), one can equivalently write equation $n+j$ in system (1.3) as

$$
\alpha_{j} \beta_{j}^{\prime} B_{n}=P_{a_{j \perp}} \sum_{k=1}^{n} A_{j+1, k} B_{n-k}+P_{a_{j \perp}} C_{j+1, n} ;
$$

this has the same format as $(2.2)$ and implies that $\beta_{j}^{\prime} B_{n}=\bar{\alpha}_{j}^{\prime} \sum_{k=1}^{n} A_{j+1, k} B_{n-k}+$ $\bar{\alpha}_{j}^{\prime} C_{j+1, n}$, and hence one has proved the following result. 
TheOREM 3.3 (Projection of Laurent coefficients). One has for any $j$

$$
P_{\beta_{j}} B_{n}=\bar{\beta}_{j} \bar{\alpha}_{j}^{\prime} \sum_{k=1}^{n} A_{j+1, k} B_{n-k}+\bar{\beta}_{j} \bar{\alpha}_{j}^{\prime} C_{j+1, n}
$$

When $\alpha_{j}, \beta_{j}$ are $0,(3.8)$ gives simply $0=0$; hence the interesting cases are for $\alpha_{j}, \beta_{j}$ different from 0 . Observe also that in (3.8) the projection $P_{\beta_{j}} B_{n}$ is expressed in terms of $B_{n-1}, \ldots, B_{0}$ and the quantities defined in (3.2), (3.4), and (3.6).

Next, one can show that the rank factorizations in (3.6) deliver complete bases of $\mathbb{C}^{p}$, namely $\left(\alpha_{0}, \ldots, \alpha_{s}\right)$ and $\left(\beta_{0}, \ldots, \beta_{s}\right)$, when $s=m$; this ensures that $\sum_{j=0}^{m} P_{\alpha_{j}}=$ $\sum_{j=0}^{m} P_{\beta_{j}}=I$ hold. Each Laurent coefficient $B_{n}$ can thus be expressed as the sum of $m+1$ orthogonal projections, $B_{n}=P_{\beta_{0}} B_{n}+\cdots+P_{\beta_{m}} B_{n}$, and subsequently reconstructed using (3.8). In what follows, $\mathbb{N}=\{1,2, \ldots\}$ is the set of positive integers.

TheOrem 3.4 (Rank conditions and order of the pole). The following statements are equivalent:

(i) $\quad A(z)^{-1}$ has a pole of order $m$, with $m \in \mathbb{N}$, at $z_{0}$;

(ii) $\quad\left(P_{a_{j \perp}} A_{j, 1} P_{b_{j \perp}}\right) B_{0}=\left\{\begin{array}{cl}0 & \text { for } j=0,1, \ldots, m-1, \\ P_{a_{m \perp}} & \text { for } j=m ;\end{array}\right.$

(iii) $\begin{cases}r_{j}<r_{j}^{\max } & \text { (reduced rank condition) for } j=0,1, \ldots, m-1, \\ r_{m}=r_{m}^{\max } & \text { (full rank condition) for } j=m ;\end{cases}$

(iv) $\quad \operatorname{col}\left(\alpha_{0}, \ldots, \alpha_{m}\right)=\operatorname{col}\left(\beta_{0}, \ldots, \beta_{m}\right)=\mathbb{C}^{p}$.

Condition (iv), together with the block-orthogonality of $\left\{\alpha_{j}\right\}$ and $\left\{\beta_{j}\right\}$, implies the projection identities

$$
I=\sum_{j=0}^{m} P_{\alpha_{j}}=\sum_{j=0}^{m} P_{\beta_{j}} .
$$

Proof. (i) $\Rightarrow$ (ii). Using (3.7), write equation $j$ in system (1.3) as $\alpha_{j} \beta_{j}^{\prime} B_{0}=$ $P_{a_{j \perp}} C_{j+1,0}$; applying definition (3.4), it is straightforward to verify that

$$
C_{s, k}=\left\{\begin{array}{cc}
0 & \text { for } s+k<m+1 \\
-I & \text { for } s+k=m+1
\end{array}\right.
$$

which implies (3.9) via (3.1).

(ii) $\Rightarrow$ (i). (3.9) implies that the identity is on the RHS of equation $m$ in (1.3), which shows that the order of the pole of the inverse is equal to $m$.

(ii) $\Rightarrow$ (iii). Substituting (3.1) in (3.9) one finds

$$
-\alpha_{j} \beta_{j}^{\prime} B_{0}=\left\{\begin{array}{cl}
0 & \text { for } j=0,1, \ldots, m-1, \\
P_{a_{m \perp}} & \text { for } j=m,
\end{array}\right.
$$

which implies $P_{\beta_{j}} B_{0}=0$ for $j=0,1, \ldots, m-1$. If the full rank condition in (3.10) held for some $j=s<m$, one would have $I=P_{\beta_{0}}+\cdots+P_{\beta_{s}}$ and hence $B_{0}=0$, which would give a contradiction. This shows that for $j=1, \ldots, m-1, r_{j}$ must be less than $r_{j}^{\max }$; i.e., the sequence of reduced rank conditions in (3.9) holds. For $j=m$, because $\operatorname{rank} P_{a_{m \perp}}=\operatorname{rank} P_{b_{m \perp}}=r_{m}^{\max }$, one has $\operatorname{rank}\left(P_{a_{m \perp}} A_{m, 1} P_{b_{m \perp}}\right) \leq r_{m}^{\max }$ and from (3.9) one finds that $P_{a_{m} \perp} A_{m, 1} P_{b_{m \perp}}$ has maximal rank $r_{m}^{\max }$; this proves (3.10). 
(iii) $\Rightarrow$ (ii). Rewrite the LHS of equation $j$ in (1.3) as $\alpha_{j} \beta_{j}^{\prime} B_{0}$ as above. The RHS of this rewriting of equation $j$ is either of rank $r_{j}^{\max }$ or of rank 0 . When $r_{j}<r_{j}^{\max }$, the RHS of equation $j$ needs to be 0 , and this happens for $j<m$. When $r_{j}=r_{j}^{\max }$, one must have hit the equation in (1.3) with the identity on the RHS; this happens when $j=m$. This implies (3.9).

(iii) $\Rightarrow$ (iv). By the definition of $r_{m}^{\max }$, one has $\sum_{j=0}^{m} r_{j}=p$; moreover, because $\left\{\beta_{j}\right\}$ are linearly independent due to block-orthogonality, this implies (3.11). A similar proof applies replacing $\left\{\beta_{j}\right\}$ with $\left\{\alpha_{j}\right\}$.

(iv) $\Rightarrow$ (iii). If $\operatorname{col}\left(\beta_{0}, \ldots, \beta_{m}\right)=\mathbb{C}^{p}$, then $\sum_{j=0}^{m} r_{j}=p$, which implies (3.10).

Theorems 3.3 and 3.4 allow one to build the recursive formula for the Laurent coefficients, as shown by the following result.

Theorem 3.5 (Laurent coefficients via extended local rank factorization). The Laurent coefficients satisfy the recursion

$$
B_{n}=C_{n}+\sum_{k=1}^{n} D_{k} B_{n-k}, \quad C_{n}:=\sum_{j=0}^{m} \bar{\beta}_{j} \bar{\alpha}_{j}^{\prime} C_{j+1, n}, \quad D_{k}:=\sum_{j=0}^{m} \bar{\beta}_{j} \bar{\alpha}_{j}^{\prime} A_{j+1, k},
$$

where $A_{s, k}, C_{s, k}$ are defined in (3.2), (3.4) and $\alpha_{j}, \beta_{j}$ in (3.6); note that $C_{0}=$ $-\bar{\beta}_{m} \bar{\alpha}_{m}^{\prime}$.

Proof. Using (3.11), one has $B_{n}=\sum_{j=0}^{m} P_{\beta_{j}} B_{n}$; substituting (3.8) in the last expression and rearranging, one finds (3.13).

Corollary 3.6 (Simplifications of the Laurent coefficients). One can rewrite (3.13) as

$$
B_{n}=\left\{\begin{array}{cc}
-\bar{\beta}_{m} \bar{\alpha}_{m}^{\prime} & \text { if } n=0, \\
C_{n}+\sum_{k=1}^{g} D_{k} B_{n-k} & \text { if } 1 \leq n \leq m, \\
\sum_{k=1}^{g} D_{k} B_{n-k} & \text { otherwise, }
\end{array} \quad g=: \min (n, \operatorname{deg} A(z))\right.
$$

When $A(z)$ is a matrix function, i.e., $\operatorname{deg} A(z)=\infty$, the sums in (3.14) involve $n$ terms; when $A(z)$ is a matrix polynomial, i.e., $\operatorname{deg} A(z)<\infty$, those sums involve at most $\operatorname{deg} A(z)$ terms.

Proof. As a direct consequence of (3.12), applying the definition of $C_{n}$ in (3.13) one finds that $C_{0}=-\bar{\beta}_{m} \bar{\alpha}_{m}^{\prime}$. Moreover, note that if $A(z)$ is a matrix polynomial of degree $d$, i.e., $A_{k}=0$ for $k>d$, then definition (3.2) implies $A_{s, k}=0$ for $k>d$, which yields $C_{s, k}=0$ for $k>d$ and hence $C_{n}=0$ for $n \geq d+1$. Finally, observe that definition (3.4) implies $C_{s, k}=0$ for $k>m$, which yields $C_{n}=0$ for $n \geq m+1$. Collecting these facts together, one can rewrite (3.13) as (3.14).

4. Local Smith form, extended canonical system of root functions, and Jordan pairs. This section links $\left\{\alpha_{j}, \beta_{j}, r_{j}, A_{j, k}\right\}$ with the local Smith form of $A(z)$ at $z_{0}$, its extended canonical systems of root functions, and Jordan pairs; see [13] for their definitions. In particular, it is shown that the values of $j$ with $r_{j}>0$ provide the distinct partial multiplicities of $A(z)$ at $z_{0}$ and $r_{j}$ gives the number of partial multiplicities that are equal to a given $j$. Three main results are presented: Theorems 4.2 and 4.3 provide a characterization of the local Smith form of $A(z)$ at $z_{0}$ and a construction of an extended canonical system of root functions. As a direct consequence of them, Corollary 4.4 provides a construction of Jordan pairs of $A(z)$ at $z_{0}$.

The following notation is instrumental to state these results. 
Definition 4.1 (Index sets $\mathcal{J}, \mathcal{J}_{+}$, and $\left.\mathcal{K}\right)$. Let $\mathcal{J}:=\left(j: r_{j}>0\right)$ be the ordered set that contains the $d:=\# \mathcal{J}$ indexes $j$ that correspond to nonzero ranks $r_{j}$. Indicate the elements of $\mathcal{J}$ by $\left(j_{1}, j_{2}, \ldots, j_{d}\right)$, and fix the reverse ordering $m=j_{1}>j_{2}>\cdots>$ $j_{d-1}>j_{d}=0$. Next, let $\mathcal{J}_{+}$be the ordered set that contains only the positive elements of $\mathcal{J}$, i.e.,

$$
\mathcal{J}_{+}:=(j \in \mathcal{J}: j>0)=\left(j_{1}, j_{2}, \ldots, j_{d-1}\right) .
$$

Finally, let $\mathcal{K}$ be the ordered set that contains each $j \in \mathcal{J}_{+}$repeated $r_{j}$ times and indicate its elements by $\left(k_{1}, k_{2}, \ldots, k_{p-r_{0}}\right):=\mathcal{K}$, i.e.,

$$
\begin{aligned}
\mathcal{K} & :=(\underbrace{j_{1}, \ldots, j_{1}}_{r_{j_{1}} \text { times }}, \underbrace{j_{2}, \ldots, j_{2}}_{r_{j_{2}} \text { times }}, \ldots, \underbrace{j_{d-1}, \ldots, j_{d-1}}_{r_{j_{d-1}} \text { times }}) \\
& =(\underbrace{k_{1}, \ldots, k_{r_{j_{1}}}}_{\text {equal to } j_{1}}, \underbrace{k_{r_{j_{1}}+1}, \ldots, k_{r_{j_{1}}+r_{j_{2}}}}_{\text {equal to } j_{2}}, \ldots \underbrace{k_{\sum_{i=1}^{d-2} r_{j_{i}}+1}, \ldots, k_{p-r_{0}}}_{\text {equal to } j_{d-1}}) .
\end{aligned}
$$

Note that $\mathcal{J}=\left(\mathcal{J}_{+}, 0\right)$ and that the index set $\mathcal{J}_{+}$contains at least one element (equal to $m$ ) and at most $m$ elements, $\mathcal{J}_{+}=(m, m-1, \ldots, 1)$, i.e., $1 \leq d-1 \leq m$. The index set $\mathcal{K}$ contains $\sum_{j \in \mathcal{J}_{+}} r_{j}=p-r_{0}$ elements. In the following, $\left(a_{j}\right)_{j \in \mathcal{J}}$ indicates $\left(a_{m}, \ldots, a_{0}\right)$ and $\operatorname{diag}\left(a_{j}\right)_{j \in \mathcal{J}}$ indicates a block diagonal matrix with $a_{m}, \ldots, a_{0}$ on the main diagonal.

THEOREM 4.2 (Local Smith form and an extended canonical system of root functions). Define the $p \times r_{j}$ matrix function

$$
\gamma_{j}(z):=\left(\beta_{j}^{\prime}-\bar{\alpha}_{j}^{\prime} \sum_{k=1}^{m-j-1} A_{j+1, k}\left(z-z_{0}\right)^{k}\right)^{\prime}, \quad j \in \mathcal{J},
$$

and the $p \times p$ matrix functions

$$
\begin{aligned}
& \Gamma(z):=\left(\gamma_{j}(z)\right)_{j \in \mathcal{J}}^{\prime}=\left(\begin{array}{c}
\gamma_{m}(z)^{\prime} \\
\vdots \\
\gamma_{0}(z)^{\prime}
\end{array}\right), \\
& \Lambda(z):=\operatorname{diag}\left(\left(z-z_{0}\right)^{j} I_{r_{j}}\right)_{j \in \mathcal{J}}=\left(\begin{array}{lll}
\left(z-z_{0}\right)^{m} I_{r_{m}} & \\
& \ddots & \\
& & \left(z-z_{0}\right)^{0} I_{r_{0}}
\end{array}\right)
\end{aligned}
$$

Then

$$
A(z) \Phi(z)=\widetilde{\Phi}(z) \Lambda(z), \quad \Phi(z):=\Gamma(z)^{-1}, \quad \operatorname{det} \Phi\left(z_{0}\right) \neq 0, \quad \operatorname{det} \widetilde{\Phi}\left(z_{0}\right) \neq 0 ;
$$

i.e., $\Lambda(z)$ is the local Smith form of $A(z)$ at $z_{0}$, and $\Phi(z)$ is an extended canonical system of root functions of $A(z)$ at $z_{0}$.

Proof. The proof proceeds as follows: first one shows that

$$
\gamma_{j}(z)^{\prime} A(z)^{-1}=\left(z-z_{0}\right)^{-j} \widetilde{\gamma}_{j}(z)^{\prime}, \quad j \in \mathcal{J}, \quad \widetilde{\gamma}_{j}\left(z_{0}\right) \neq 0,
$$

where $\gamma_{j}(z)$ is as defined in (4.1). Then one can rewrite (4.4) as $\Gamma(z) A(z)^{-1}=$ $\Lambda(z)^{-1} \widetilde{\Gamma}(z)$, where $\Gamma(z), \Lambda(z)$ are defined in (4.2) and $\widetilde{\Gamma}(z):=\left(\widetilde{\gamma}_{j}(z)\right)_{j \in \mathcal{J}}^{\prime}$, and finally one can show that $\Gamma\left(z_{0}\right), \widetilde{\Gamma}\left(z_{0}\right)$ are nonsingular. This implies that $A(z) \Gamma(z)^{-1}=$ 
$\widetilde{\Gamma}(z)^{-1} \Lambda(z)$, and hence (4.3) holds. Thus (see Theorems 1.2 and 1.3 in [13]), $\Lambda(z)$ is the local Smith form of $A(z)$ at $z_{0}$ and $\Phi(z)$ is an extended system of root functions of $A(z)$ at $z_{0}$.

One hence starts by proving (4.4); write (1.2) as

$A(z)^{-1}=B_{0}\left(z-z_{0}\right)^{-m}+\sum_{n=1}^{m-j-1} B_{n}\left(z-z_{0}\right)^{n-m}+\left(z-z_{0}\right)^{-j} R_{0}(z), \quad R_{0}\left(z_{0}\right)=B_{m-j}$,

and premultiply it by $\beta_{j}^{\prime}$ to find that

$$
\beta_{j}^{\prime} A(z)^{-1}=\beta_{j}^{\prime} B_{0}\left(z-z_{0}\right)^{-m}+\sum_{n=1}^{m-j-1} \beta_{j}^{\prime} B_{n}\left(z-z_{0}\right)^{n-m}+\left(z-z_{0}\right)^{-j} \beta_{j}^{\prime} R_{0}(z) .
$$

First, consider $j \neq m$. From (3.14) one has $B_{0}=-\bar{\beta}_{m} \bar{\alpha}_{m}^{\prime}$ and hence $\beta_{j}^{\prime} B_{0}=0$; then

$$
\beta_{j}^{\prime} A(z)^{-1}=\sum_{n=1}^{m-j-1} \beta_{j}^{\prime} B_{n}\left(z-z_{0}\right)^{n-m}+\left(z-z_{0}\right)^{-j} \beta_{j}^{\prime} R_{0}(z)=: U(z)+V(z),
$$

where $U(z)$ is the first sum after the equal sign and

$$
\beta_{j}^{\prime} B_{n}=\bar{\alpha}_{j}^{\prime} \sum_{k=1}^{n} A_{j+1, k} B_{n-k}+\bar{\alpha}_{j}^{\prime} C_{j+1, n}
$$

follows from (3.7). Observe that for $n=1, \ldots, m-j-1$ one has $j+1+n \leq m$, and hence $C_{j+1, n}=0$ follows from (3.12); substituting (4.6) and rearranging terms, one can rewrite $U(z)$ as

$$
\begin{aligned}
U(z) & =\sum_{n=1}^{m-j-1}\left(\bar{\alpha}_{j}^{\prime} \sum_{k=1}^{n} A_{j+1, k} B_{n-k}\right)\left(z-z_{0}\right)^{n-m} \\
& =\bar{\alpha}_{j}^{\prime} \sum_{k=1}^{m-j-1} A_{j+1, k}\left(\sum_{n=k}^{m-j-1} B_{n-k}\left(z-z_{0}\right)^{n-m}\right) .
\end{aligned}
$$

Next, multiply (1.2) by $\left(z-z_{0}\right)^{k}$ and write it as

$$
\left(z-z_{0}\right)^{k} A(z)^{-1}=\left(\sum_{n=k}^{m-j-1} B_{n-k}\left(z-z_{0}\right)^{n-m}\right)+\left(z-z_{0}\right)^{-j} R_{k}(z), \quad R_{k}\left(z_{0}\right)=B_{m-j-k} ;
$$

then

$$
\begin{aligned}
& U(z)=\left(\bar{\alpha}_{j}^{\prime} \sum_{k=1}^{m-j-1} A_{j+1, k}\left(z-z_{0}\right)^{k}\right) A(z)^{-1}-\left(z-z_{0}\right)^{-j} \bar{\alpha}_{j}^{\prime} \sum_{k=1}^{m-j-1} A_{j+1, k} R_{k}(z), \\
& U(z)+V(z)=\left(\bar{\alpha}_{j}^{\prime} \sum_{k=1}^{m-j-1} A_{j+1, k}\left(z-z_{0}\right)^{k}\right) A(z)^{-1} \\
& +\left(z-z_{0}\right)^{-j}\left(\beta_{j}^{\prime} R_{0}(z)-\bar{\alpha}_{j}^{\prime} \sum_{k=1}^{m-j-1} A_{j+1, k} R_{k}(z)\right),
\end{aligned}
$$


and (4.5) is rewritten as

$$
\gamma_{j}(z)^{\prime} A(z)^{-1}=\left(z-z_{0}\right)^{-j} \widetilde{\gamma}_{j}(z)^{\prime}
$$

where $\gamma_{j}(z)$ is defined in (4.1) and

$$
\widetilde{\gamma}_{j}(z):=\left(\beta_{j}^{\prime} R_{0}(z)-\bar{\alpha}_{j}^{\prime} \sum_{k=1}^{m-j-1} A_{j+1, k} R_{k}(z)\right)^{\prime} .
$$

Note that

$$
\gamma_{j}\left(z_{0}\right)=\beta_{j}, \quad \widetilde{\gamma}_{j}\left(z_{0}\right)=\left(\beta_{j}^{\prime} B_{m-j}-\bar{\alpha}_{j}^{\prime} \sum_{k=1}^{m-j-1} A_{j+1, k} B_{m-j-k}\right)^{\prime},
$$

where the last expression follows from $R_{k}\left(z_{0}\right)=B_{m-j-k}$. Now consider (3.3) for $n=m$ and write

$$
\beta_{j}^{\prime} B_{m-j}=\bar{\alpha}_{j}^{\prime} \sum_{k=1}^{m-j} A_{j+1, k} B_{m-j-k}+\bar{\alpha}_{j}^{\prime} C_{j+1, m-j} ;
$$

because $C_{j+1, m-j}=-I$ (see $\left.(3.12)\right)$, one then finds that

$$
\widetilde{\gamma}_{j}\left(z_{0}\right)^{\prime}=\bar{\alpha}_{j}^{\prime} A_{j+1, m-j} B_{0}-\bar{\alpha}_{j}^{\prime}=-\bar{\alpha}_{j}^{\prime} A_{j+1, m-j} \bar{\beta}_{m} \bar{\alpha}_{m}^{\prime}-\bar{\alpha}_{j}^{\prime}
$$

where the last expression follows from $B_{0}=-\bar{\beta}_{m} \bar{\alpha}_{m}^{\prime}$. This implies $\widetilde{\gamma}_{j}\left(z_{0}\right)^{\prime} \alpha_{j}=-I_{r_{j}}$, and thus it proves that (4.4) holds for $j \neq m$.

Now consider $j=m$. From (3.14) one has $\beta_{m}^{\prime} B_{0}=-\bar{\alpha}_{m}^{\prime} \neq 0$ and hence $\gamma_{m}(z)^{\prime} A(z)^{-1}=\left(z-z_{0}\right)^{-m} \widetilde{\gamma}_{m}(z)^{\prime}$, where $\gamma_{m}(z):=\beta_{m}$ and $\widetilde{\gamma}_{m}\left(z_{0}\right)=-\bar{\alpha}_{m} \neq 0$. This completes the proof of (4.4). Finally, one can show that $\Gamma\left(z_{0}\right), \widetilde{\Gamma}\left(z_{0}\right)$ are nonsingular. Because $\Gamma\left(z_{0}\right)=\left(\beta_{j}\right)_{j \in \mathcal{J}}^{\prime}$, the first statement follows from (3.11). Because $\widetilde{\gamma}_{j}\left(z_{0}\right)^{\prime} \alpha_{j}=-I_{r_{j}}$ and $\widetilde{\gamma}_{j}\left(z_{0}\right)^{\prime} \alpha_{h}=0$ for $h<j \in \mathcal{J}, \widetilde{\Gamma}\left(z_{0}\right)\left(\alpha_{j}\right)_{j \in \mathcal{J}}$ is a lower triangular matrix with identities on the main diagonal, and hence it is nonsingular. Because $\left(\alpha_{j}\right)_{j \in \mathcal{J}}$ is nonsingular (see (3.11)), the same holds for $\widetilde{\Gamma}\left(z_{0}\right)$.

Theorem 4.2 links the rank structure of $A(z)$ at $z_{0}$, derived in Lemma 3.1 and Theorem 3.4, to the local spectral characteristics of $A(z)$ at $z_{0}$. In particular, it delivers the structure of the local Smith form, $\Lambda(z)$, and provides an extended canonical system of root functions, $\Phi(z)$. From $\Lambda(z)$ in (4.2) one sees that the distinct partial multiplicities of $A(z)$ at $z_{0}$ are equal to the elements of $\mathcal{J}$ and further that there are exactly $r_{j}$ partial multiplicities for each $j \in \mathcal{J}$. Note that $\mathcal{J}_{+}$contains the nonzero partial multiplicities; information on them is also presented in the index set $\mathcal{K}$, which lists them with the corresponding repetitions. That is, full information on the orders and the number of root functions of the same nonzero order in an extended canonical system is available given $\mathcal{K}$.

The next result expresses the coefficients of $\Phi(z)$ in terms of $\left\{\alpha_{j}, \beta_{j}, r_{j}, A_{j, k}\right\}$.

Theorem 4.3 (Coefficients of the extended canonical system of root functions). Write $\Phi(z)$ in $(4.2)$ as $\Phi(z)=\sum_{n=0}^{\infty} \Phi_{n}\left(z-z_{0}\right)^{n}$; then

$$
\Phi_{0}=\left(\bar{\beta}_{j}\right)_{j \in \mathcal{J}}, \quad \Phi_{n}=\sum_{k=1}^{\min (n, m-1)} D_{m-k, k} \Phi_{n-k}, \quad n \geq 1,
$$

where $D_{m-k, k}:=\sum_{j=0}^{m-k-1} \bar{\beta}_{j} \bar{\alpha}_{j}^{\prime} A_{j+1, k}$. 
Proof. From (4.1) one has that the degree of $\gamma_{j}(z)$ is $m-j-1$, and thus $\Gamma(z)$ in (4.2) has degree $m-1$. Writing $\Gamma(z)=\Gamma_{0}-\sum_{k=1}^{m-1} \Gamma_{k}\left(z-z_{0}\right)^{k}$, one then has

$$
\begin{gathered}
\Gamma_{0}=\left(\beta_{j}\right)_{j \in \mathcal{J}}^{\prime}=\left(\begin{array}{c}
\beta_{m}^{\prime} \\
\vdots \\
\beta_{0}^{\prime}
\end{array}\right), \quad \Gamma_{k}=\left(\begin{array}{c}
0 \\
\Psi_{k}
\end{array}\right), \\
\Psi_{k}:=\left(A_{j+1, k}^{\prime} \bar{\alpha}_{j}\right)_{j \in \mathcal{J}_{k}}^{\prime}=\left(\begin{array}{c}
\bar{\alpha}_{m-k-1}^{\prime} A_{m-k, k} \\
\vdots \\
\bar{\alpha}_{0}^{\prime} A_{1, k}
\end{array}\right),
\end{gathered}
$$

where $\mathcal{J}_{k}:=\{j \in \mathcal{J}: j \leq m-k-1\}$ and in the last equality it is assumed that $m-k-1$ belongs to $\mathcal{J}$.

Using the identity $\Gamma(z) \Phi(z)=I$, one finds that

$$
\Phi_{0}=\Gamma_{0}^{-1}, \quad \Phi_{n}=\Gamma_{0}^{-1} \sum_{k=1}^{\min (n, m-1)} \Gamma_{k} \Phi_{n-k}, \quad n \geq 1 ;
$$

since $\Gamma_{0}^{-1}=\left(\bar{\beta}_{j}\right)_{j \in \mathcal{J}}$, one has $\Gamma_{0}^{-1} \Gamma_{k}=\sum_{j \in \mathcal{J}_{k}} \bar{\beta}_{j} \bar{\alpha}_{j}^{\prime} A_{j+1, k}$ and thus the statement.

Observe that even though the coefficients of $\Phi(z)$ depend on the choice of bases in the rank factorizations, any other choice delivers an alternative extended canonical system of root functions.

Given $\Phi(z)$ and $\mathcal{K}$, one can construct a Jordan pair of $A(z)$ at $z_{0}$ as follows.

Corollary 4.4 (Jordan pair). Let $\phi_{i, n}$ be the ith column of $\Phi_{n}$, and let $k_{i}$ be the ith element in the index set $\mathcal{K}$; for $i=1, \ldots, p-r_{0}$, define

$$
X_{i}:=\left(\phi_{i, n}\right)_{n=0}^{k_{i}-1}, \quad J_{k_{i}}:=\left(\begin{array}{cccc}
z_{0} & 1 & & \\
& \ddots & \ddots & \\
& & z_{0} & 1 \\
& & & z_{0}
\end{array}\right)
$$

respectively, of dimensions $p \times k_{i}$ and $k_{i} \times k_{i}$. Then the columns of $X_{i}$ form a Jordan chain of maximal length $k_{i}$ and $J_{k_{i}}$ is the corresponding Jordan block. Collecting the Jordan chains and the Jordan blocks, respectively, in

$$
X:=\left(X_{i}\right)_{i=1}^{p-r_{0}}, \quad J:=\operatorname{diag}\left(I_{r_{j}} \otimes J_{j}\right)_{j \in \mathcal{J}_{+}},
$$

one has that $(X, J)$ is a Jordan pair of $A(z)$ at $z_{0}$.

Proof. The proof is a direct consequence of Theorems 4.2 and 4.3 and the definition of Jordan pairs in [13].

5. Algorithmic implementation. This section contains an algorithmic implementation of the ELRF. This is formulated in Definition 5.3. A MATLAB script that implements the ELRF is provided in the supplementary material (99983_01.pdf [local/web 162KB]). The connections with the CRP in [3] are discussed in Theorem 5.10, where it is shown that the CRP coincides with the ELRF, and in Corollary 5.11, which links the characteristics of the CRP to the structure of the local Smith form of $A(z)$ at $z_{0}$.

Before describing the ELRF algorithm, it is useful to discuss the implementation of the rank factorizations, which constitute its building blocks. In the following, $\{r, \xi, \eta\}$ 
are said to be given by the rank factorization of $\varphi$ when $\varphi=-\xi \eta^{\prime}$, and $\xi$ and $\eta$ are full column matrices with $r$ columns. It is also assumed that $\bar{\xi}, \bar{\eta}, \xi_{\perp}, \eta_{\perp}, \bar{\xi}_{\perp}, \bar{\eta}_{\perp}$ can be simultaneously computed, as illustrated in the following remarks.

Remark 5.1 (Rank factorization via SVD). Several standard matrix procedures can be used to perform the rank factorization $\{r, \xi, \eta\}$ of $\varphi$; here computations are illustrated using the singular value decomposition (SVD); see, e.g., [18]. Let $\varphi=$ $U S V^{\prime}$ represent the SVD of $\varphi$, where $U^{\prime} U=V^{\prime} V=I$ and $S=\operatorname{diag}\left(s_{1}^{2}, \ldots, s_{p}^{2}\right)$, with $s_{1}^{2} \geq \cdots \geq s_{p}^{2} \geq 0$. The rank of $\varphi$ is numerically computed as the largest integer $r$ for which $s_{1}^{2} \geq \cdots \geq s_{r}^{2}>0$ and $s_{r+1}^{2}=\cdots=s_{p}^{2}=0$. Given $r$, one can define $\xi=-U_{1}, \eta=V_{1} S_{1}, \xi_{\perp}=U_{2}, \eta_{\perp}=V_{2}$, where $U=\left(U_{1}, U_{2}\right)$ and $V=\left(V_{1}, V_{2}\right)$ are partitioned into blocks of the first $r$ columns (with subscript 1) and the last $p-r$ columns (with subscript 2), and $S_{1}=\operatorname{diag}\left(s_{1}^{2}, \ldots, s_{r}^{2}\right)$. With this choice, one has $\xi^{\prime} \xi=I_{r}, \xi_{\perp}^{\prime} \xi_{\perp}=\eta_{\perp}^{\prime} \eta_{\perp}=I_{p-r}$ so that $\bar{\xi}=\xi, \bar{\xi}_{\perp}=\xi_{\perp}, \bar{\eta}_{\perp}=\eta_{\perp}$; that is, no matrix inversion is involved when computing the "bar" operation $\bar{\zeta}:=\zeta\left(\zeta^{\prime} \zeta\right)^{-1}$ in these cases. Moreover, one has $\bar{\eta}=V_{1} \operatorname{diag}\left(s_{1}^{-2}, \ldots, s_{r}^{-2}\right)$. This requires the inversion of the diagonal matrix $S_{1}$, which can be computed elementwise. Note that this is one possible choice of bases of the various spaces; this specific choice is convenient because the only matrix inversion involved can be performed elementwise.

Remark 5.2 (Rank factorization via QR). As an alternative to the SVD, one can consider using the QR decomposition; see, e.g., [18]. Let $\varphi=Q R$ represent the QR decomposition of $\varphi$, where $Q^{\prime} Q=Q Q^{\prime}=I$ and $R$ is upper triangular. The rank $r$ of $\varphi$ is numerically computed as the number of nonzero rows of $R$. Given $r$, one can define $\xi=-Q_{1}, \xi_{\perp}=Q_{2}, \eta=R_{1}$, where $Q=\left(Q_{1}, Q_{2}\right)$ and $R^{\prime}=\left(R_{1}, R_{2}\right)$ are partitioned into blocks of the first $r$ columns (with subscript 1) and the last $p-r$ columns (with subscript 2). With this choice, one has $\xi^{\prime} \xi=I_{r}$ and $\xi_{\perp}^{\prime} \xi_{\perp}=I_{p-r}$ so that $\bar{\xi}=\xi$ and $\bar{\xi}_{\perp}=\xi_{\perp}$. However, unlike the SVD, the QR decomposition does not return directly $\eta_{\perp}$, which can be computed using a second QR decomposition to $\eta$, namely $\eta=Q^{\circ} R^{\circ}$. With this choice, one has $\eta_{\perp}=Q_{2}^{\circ}$, where $Q^{\circ}=\left(Q_{1}^{\circ}, Q_{2}^{\circ}\right)$ is partitioned into blocks of the first $r$ columns and the last $p-r$ columns, which also satisfies $\eta_{\perp}^{\prime} \eta_{\perp}=I_{p-r}$ so that $\bar{\eta}_{\perp}=\eta_{\perp}$. Hence, the same output obtained via the SVD can be computed from the QR decomposition, provided this is applied twice.

The next definition contains an algorithmic implementation of the ELRF.

DEFINITION 5.3 (The ELRF algorithm).

INPUT: The inputs are the $p \times p$ matrices $\left\{A_{n}\right\}_{n=0}^{\infty}$ and the number $q$ of Laurent coefficients $B_{0}, \ldots, B_{q-1}$ to be computed. ${ }^{2}$

OutPut: The outputs are the scalar $m$ and the Laurent coefficients $B_{0}, \ldots, B_{q-1}$. Initialization: Set $j=0, r_{0}^{\max }=p, \mathcal{J}_{0}=0$, and $a_{0}=b_{0}=0$. Compute $\left\{r_{0}, \xi_{0}, \eta_{0}\right\}$ as the rank factorization of $A_{0}$,

$$
A_{0}=-\xi_{0} \eta_{0}^{\prime}
$$

and set $a_{1}=\alpha_{0}=\xi_{0}, b_{1}=\beta_{0}=\eta_{0}, \theta_{0}=\bar{\beta}_{0} \bar{\alpha}_{0}^{\prime}$. Go to RECURSION.

RECURSION: If $r_{j}=r_{j}^{\max }$, then go to FINAL LOOPS; else increase $j$ by 1 and perform all the following computations: Set $r_{j}^{\max }=r_{j-1}^{\max }-r_{j-1}$, compute $A_{1, j}=A_{j}$ and $D_{1, j}=\theta_{0} A_{1, j}$, and for $s=2, \ldots, j$ compute $A_{s, j-s+1}$ and $D_{s, j-s+1}$ using

$$
A_{s, k}=A_{s-1, k+1}+A_{s-1,1} D_{s-1, k}, \quad D_{s, k}=D_{s-1, k}+\theta_{s-1} A_{s, k} .
$$

\footnotetext{
${ }^{2}$ Because $A_{j}$ is used in RECURSIOn $j$, in practice only a finite number of $A_{n}$ matrices is needed as input to the algorithm.
} 
Next, calculate $\left\{r_{j}, \xi_{j}, \eta_{j}\right\}$ as the rank factorization of $a_{j \perp}^{\prime} A_{j, 1} b_{j \perp}$,

$$
a_{j \perp}^{\prime} A_{j, 1} b_{j \perp}=-\xi_{j} \eta_{j}^{\prime}
$$

where $a_{j \perp}=a_{j-1 \perp} \xi_{j-1 \perp}$ and $b_{j \perp}=b_{j-1 \perp} \eta_{j-1 \perp}$. If $r_{j}=0$, define $\mathcal{J}_{j}=\mathcal{J}_{j-1}$, $a_{j+1}=a_{j}, b_{j+1}=b_{j}$, and $\theta_{j}=0$; else (i.e., $1 \leq r_{j} \leq r_{j}^{\max }$ ) set $\mathcal{J}_{j}=\left(j, \mathcal{J}_{j-1}\right)$, $\alpha_{j}=\bar{a}_{j \perp} \xi_{j}, \beta_{j}=\bar{b}_{j \perp} \eta_{j}, \theta_{j}=\bar{\beta}_{j} \bar{\alpha}_{j}^{\prime}, a_{j+1}=\left(\alpha_{j}, a_{j}\right)$, and $b_{j+1}=\left(\beta_{j}, b_{j}\right)$.

FINAL LOOPS: Set $m=j, \mathcal{J}=\mathcal{J}_{j}, a=a_{j+1}, b=b_{j+1}$ and compute $D_{m+1, k}$ using (5.2) for $k=1, \ldots, q-1$. Next, let $C_{1, k}=-\delta_{k, m} I, E_{1, k}=-\theta_{0} \delta_{k, m}$ and compute $E_{m+1, k}$ for $k=0, \ldots, q-1$ using the following recursions to compute $E_{s, m+1+k-s}$ for $s=2, \ldots, m+1$ :

$$
C_{s, k}=C_{s-1, k+1}+A_{s-1,1} E_{s-1, k}, \quad E_{s, k}=E_{s-1, k}+\theta_{s-1} C_{s, k} .
$$

Finally, use (3.14) to compute $B_{n}, n=0, \ldots, q-1$, with $C_{k}=E_{m+1, k}, D_{k}=D_{m+1, k}$.

A few remarks are in order.

Remark 5.4 (Extension). The recursion of the ELRF coincide with the LRF of [10]; the extension refers to the final loops, which allow one to calculate the Laurent coefficients $B_{n}, n=1, \ldots, q-1$.

Remark 5.5 (Definitions). The matrices in (5.1), (5.2), (5.3), and (5.4) coincide with those defined in Lemma 3.1 and Theorem 4.3.

Remark 5.6 (Order of the pole). The ELRF algorithm determines the order of the pole $m$ by checking the ranks of the $r_{j}^{\max } \times r_{j}^{\max }$ matrices $a_{j \perp}^{\prime} A_{j, 1} b_{j \perp}$ in (5.3) until full rank is found. This stopping condition terminates the recursion and determines the order of the pole. This exploits the results in Theorem 3.4.

Remark 5.7 (Dimension of rank factorizations). Successive rank decompositions are performed on matrices of nonincreasing dimension, i.e., $r_{j}^{\max } \leq r_{j-1}^{\max } \leq p-r_{0}$, where $p$ is the dimension of $A_{n}$ and $r_{0}$ is the rank of $A_{0}$.

Remark 5.8 (Nonuniqueness of factors in rank factorizations). Because in a rank decomposition the factors are not unique, one of them can be chosen to be orthonormal, for instance the first one as in Remark 5.1. In this case, one has $\xi_{j}=\bar{\xi}_{j}$ $\left(\alpha_{j}=\bar{\alpha}_{j}\right)$ so that only $\bar{\eta}_{j}\left(\bar{\beta}_{j}\right)$ needs to be computed. Similarly, because $\xi_{j \perp}, \eta_{j \perp}$ are any bases of the orthogonal complements of $\operatorname{col} \xi_{j}$ and $\operatorname{col} \eta_{j}$, one can choose them to be orthonormal. In this case, because $a_{j \perp}$ can also be chosen orthonormal, one finds that $a_{j+1 \perp}=a_{j \perp} \xi_{j \perp}$. Similar remarks apply to $\eta_{j \perp}$ and $b_{j \perp}$.

Despite the nonuniqueness of factors in the rank factorizations, the outputs of the ELRF are invariant with respect to the choice of bases. Hence, in general, this nonuniqueness can be exploited in an advantageous way for subsequent calculations.

Remark 5.9 (The ELRF and Moore-Penrose inverses). Note that (5.2) and (5.4) coincide with (3.2) and (3.4). These expressions involve Moore-Penrose inverses of dimension $p$ because $\bar{\beta}_{j} \bar{\alpha}_{j}^{\prime}$ is the Moore-Penrose inverse of $\alpha_{j} \beta_{j}^{\prime}$; see, e.g., Theorem 5 on p. 48 of [6]. However, in practice one can compute $\bar{\beta}_{j} \bar{\alpha}_{j}^{\prime}$ using Moore-Penrose inverses of matrices of dimension $r_{j}^{\max } \leq r_{j-1}^{\max } \leq p-r_{0}$; in fact, because $\bar{\alpha}_{j}=$ $a_{j \perp} \bar{\xi}_{j}$ and $\bar{\beta}_{j}=b_{j \perp} \bar{\eta}_{j}$, one has $\bar{\beta}_{j} \bar{\alpha}_{j}^{\prime}=b_{j \perp} \bar{\eta}_{j} \bar{\xi}_{j} a_{j \perp}^{\prime}$. Here $\bar{\eta}_{j} \bar{\xi}_{j}^{\prime}$ is the Moore-Penrose inverse of $\xi_{j} \eta_{j}^{\prime}$ of dimension $r_{j}^{\max }$. It can also be noted that if one performs the rank factorizations as illustrated in Remark 5.1, the only matrix inversion involved in $\bar{\eta}_{j} \bar{\xi}_{j}^{\prime}$ is that of a $r_{j}^{\max } \times r_{j}^{\max }$ diagonal matrix, which can be performed elementwise. 
Next, attention is turned to the relation between the ELRF and the CRP in [3]. Given that in Theorem 5.10 below the two procedures are shown to coincide, it follows that (3.13) provides the explicit recursive formula to compute the Laurent coefficients when the CRP is performed.

TheOREM 5.10 (CRP and ELRF). The CRP coincides with the ELRF.

Proof. Substitute $\alpha_{j}=\bar{a}_{j \perp} \xi_{j}, \beta_{j}=\bar{b}_{j \perp} \eta_{j}$ in (3.7) and premultiply by $a_{j \perp}^{\prime}$ to find that

$$
\xi_{j} \eta_{j}^{\prime} \bar{b}_{j \perp}^{\prime} B_{n}=a_{j \perp}^{\prime} \sum_{k=1}^{n} A_{j+1, k} B_{n-k}+a_{j \perp}^{\prime} C_{j+1, n} ;
$$

inserting the projection identity $I=P_{b_{j \perp}}+P_{b_{j}}$ between $A_{j+1, k}$ and $B_{n-k}$, one then finds that

$$
\xi_{j} \eta_{j}^{\prime} \bar{b}_{j \perp}^{\prime} B_{n}=\sum_{k=1}^{n} a_{j \perp}^{\prime} A_{j+1, k} P_{b_{j \perp}} B_{n-k}+\sum_{k=1}^{n} a_{j \perp}^{\prime} A_{j+1, k} P_{b_{j}} B_{n-k}+a_{j \perp}^{\prime} C_{j+1, n} .
$$

Note that (5.5) for $j=0$ gives the original system (1.3). One can next show that, given the system (5.5), the application of one reduction step in the sense of [3] leads to the next matrix rank factorization in the ELRF. This shows that the CRP coincides with the ELRF.

First, observe that (5.5) can be written in the format of equations (8.0)-(8.t) in $[3],^{3}$

$$
C_{0} V_{n}+\sum_{k=1}^{n} C_{k} V_{n-k}=R_{n}
$$

by setting $C_{0}=\xi_{j} \eta_{j}^{\prime}, C_{k}=-a_{j \perp}^{\prime} A_{j+1, k} b_{j \perp}, V_{n}=\bar{b}_{j \perp}^{\prime} B_{n}$, and

$$
R_{n}=\sum_{k=1}^{n} a_{j \perp}^{\prime} A_{j+1, k} P_{b_{j}} B_{n-k}+a_{j \perp}^{\prime} C_{j+1, n} .
$$

One can next apply a reduction step to (5.6) premultiplying it by a basis of the left null space of $C_{0}$ to find that

$$
\sum_{k=1}^{n} \xi_{j \perp}^{\prime} C_{k} V_{n-k}=\xi_{j \perp}^{\prime} R_{n}
$$

Observe that $\xi_{j \perp}^{\prime} C_{k} V_{n-k}=-\xi_{j \perp}^{\prime} a_{j \perp}^{\prime} A_{j+1, k} P_{b_{j \perp}} B_{n-k}=-a_{j+1 \perp}^{\prime} A_{j+1, k} P_{b_{j+1 \perp}} B_{n-k}-$ $a_{j+1 \perp}^{\prime} A_{j+1, k} P_{\beta_{j}} B_{n-k}$, where the last equality follows by definition from $a_{j+1 \perp}=$ $a_{j \perp} \xi_{j \perp}$ and $P_{b_{j \perp}}=P_{b_{j+1 \perp}}+P_{\beta_{j}}$. Rearranging terms and setting $s=n-1$, one has

$$
-a_{j+1 \perp}^{\prime} A_{j+1,1} P_{b_{j+1 \perp}} B_{s}-\sum_{k=1}^{s} a_{j+1 \perp}^{\prime} A_{j+1, k+1} P_{b_{j+1 \perp}} B_{s-k}=S_{s},
$$

where $S_{s}=\xi_{j \perp}^{\prime} R_{s+1}+\sum_{k=1}^{s+1} a_{j+1 \perp}^{\prime} A_{j+1, k} P_{\beta_{j}} B_{s+1-k}$. This can be rewritten in the format of equations (10.0)-(10.t-1) in [3],

$$
D_{0} W_{s}+\sum_{k=1}^{s} D_{k} W_{s-k}=S_{s}
$$

\footnotetext{
${ }^{3}$ Here and in the proof of Corollary 5.11 the letters $C$ and $D$ are used to match the notation in [3], and they do not refer to (3.13) as in the rest of the paper.
} 
where $D_{k}=-a_{j+1 \perp}^{\prime} A_{j+1, k+1} b_{j+1 \perp}, W_{s-k}=\bar{b}_{j+1 \perp}^{\prime} B_{s-k}$ for $k=0, \ldots s$, and $s=$ $0, \ldots, m-1$. Because the reduced system (5.7) is again reducible if and only if $D_{0}=a_{j+1 \perp}^{\prime} A_{j+1,1} b_{j+1 \perp}$ is of reduced rank, which is the rank condition in (5.3), this proves that the CRP coincides with the ELRF.

Finally, the characteristics of the CRP are linked to the structure of the local Smith form of $A(z)$ at $z_{0}$. In Theorem 4.2, the latter is shown to be characterized by the ELRF; via Theorem 5.10, the characteristics of the CRP in [3] are thus linked to the structure of the local Smith form.

COROLlary 5.11 (CRP and structure of the local Smith form). The CRP consists of $1 \leq d-1 \leq m$ reduction steps, where $d$ is the number of distinct partial multiplicities of $A(z)$ at $z_{0}$ (see Definition 4.1); reduction step $i=1, \ldots, d-1$ decreases the dimension of the coefficients by $r_{j_{d+1-i}}$, the number of partial multiplicities that are equal to the given value $j_{d+1-i}$.

Proof. In the proof of Theorem 5.10 it is shown that dimension of the $C_{k}$ coefficients of the reducible system (5.6) is $r_{j}^{\max } \times r_{j}^{\max }$, where $r_{j}^{\max }=p-\sum_{i=0}^{j-1} r_{i}$, and the dimension of the $D_{k}$ coefficients of the reduced system (5.7) is $r_{j+1}^{\max } \times r_{j+1}^{\max }$, where $r_{j+1}^{\max }=r_{j}^{\max }-r_{j}$ and $r_{j}=\operatorname{rank} C_{0}$. Hence a reduction occurs if and only if $r_{j}>0$, i.e., $j \in \mathcal{J}$, and the dimension of the coefficients is decreased by $r_{j}$. Because each and every $j \in \mathcal{J}$ is a partial multiplicity of $A(z)$ at $z_{0}$ and there are $r_{j}$ partial multiplicities that are equal to $j$ (see $\Lambda(z)$ in (4.2)), the statement is proved.

6. Computational complexity. In this section the computational complexity of the ELRF algorithm is discussed in terms of floating point operations (flops). Because of Theorem 5.10, this corresponds to the computational complexity of the CRP. In particular, this confirms that the flops associated to the one-step reduction process are always greater than or equal to those of the CRP.

The $A B+C$ operation, where $A, B$, and $C$ are $p \times p$ matrices, requires $O\left(p^{3}\right)$ flops; the same order of complexity holds for the rank decomposition of a $p \times p$ matrix via SVD (see, e.g., p. 18 and p. 254 in [18]) or via QR (see p. 233 in [18]). ${ }^{4}$ In each recursion, $j$ operations of the type $A B+C$ are performed to compute $A_{s, k}$ in (5.2) and the same number of $A B+C$ operations is required for $D_{s, k}$ in (5.2). Hence the total number of $A B+C$ operations is $2 \sum_{j=1}^{m} j=m(m+1)$, corresponding to $O\left(m^{2} p^{3}\right)$ flops. The total complexity of the rank decompositions is always less than $O\left(m p^{3}\right)$ flops because it consists of $O\left(p^{3}\right)$ flops for (5.1) and of $O\left(\left(r_{j}^{\max }\right)^{3}\right)$ flops for (5.3), where $r_{j}^{\max } \leq p-r_{0}$.

Next, consider the final loops; each iteration involves $A B+C$ operations to compute $A_{s, k}$ and $D_{s, k}$ in (5.2) and $C_{s, k}$ and $C_{s, k}$ in (5.4). Hence this requires $O\left(\mathrm{~m}^{2} p^{3}\right)$ flops. Because there are $q-1$ final loops, this leads to a total of $O\left((q-1) m^{2} p^{3}\right)$ flops. Summing up, it can be seen that the ELRF computes $m$ and $B_{0}, \ldots, B_{q-1}$ with $O\left(q m^{2} p^{3}\right)$ flops. Note that this complexity is determined by the $A B+C$ operations and not by the matrix rank decompositions. Note also that this estimate does not include the simplifications due to the presence of zero matrices; see Corollary 3.6.

In [3], it is shown that (for known order of the pole) the one-step reduction process computes $B_{0}$ with $O\left(\max \left\{m^{2} p^{3}, m^{3}\left(p-r_{0}\right)^{3}\right\}\right)$ flops. Hence when $\max \left\{m^{2} p^{3}, m^{3}(p-\right.$ $\left.\left.r_{0}\right)^{3}\right\}=m^{2} p^{3}$, i.e., if $m \leq p^{3} /\left(p-r_{0}\right)^{3}$, the computational complexity of the one-step reduction coincides with that of the ELRF (which, however, also provides the order

\footnotetext{
${ }^{4}$ For upper Hessenberg matrices, the flops order is lower; see [18, p. 228]. For generality, however, the rest of the section refers to the generic case of unstructured matrices.
} 
of the pole), as can be seen by setting $q=1$. When $m>p^{3} /\left(p-r_{0}\right)^{3}$, there is a computational gain in using the ELRF, i.e., the CRP, with respect to the one-step reduction process. This gain arises because stacking matrices in a potentially large system and performing a Moore-Penrose inverse on it dominates the computational complexity of the $A B+C$ operations as $m$ increases.

7. Example. This section illustrates results using a numerical example; the calculations are performed using the MATLAB implementation of the ELRF provided in the supplementary material (99983_01.pdf [local/web 162KB]). Consider the matrix polynomial

$$
A(z)=\underbrace{\left(\begin{array}{lll}
1 & 0 & 0 \\
0 & 0 & 0 \\
0 & 0 & 0
\end{array}\right)}_{A_{0}}+\underbrace{\left(\begin{array}{ccc}
0 & 0 & 0 \\
0 & -1 & 0 \\
\frac{1}{2} & 0 & 0
\end{array}\right)}_{A_{1}} z+\underbrace{\left(\begin{array}{ccc}
0 & 0 & -\frac{1}{2} \\
0 & 0 & 0 \\
\frac{1}{2} & 0 & 0
\end{array}\right)}_{A_{2}} z^{2}+\underbrace{\left(\begin{array}{ccc}
0 & 0 & -\frac{1}{2} \\
0 & 0 & 0 \\
0 & 0 & -\frac{1}{4}
\end{array}\right)}_{A_{3}} z^{3},
$$

and observe that $A(0)=A_{0}$ is singular. The ELRF at $z_{0}=0$ is used to determine the order of the pole of $A(z)^{-1}$ at $z_{0}=0$, to compute the coefficients of its principal part, and to construct the local Smith form and a Jordan pair of $A(z)$ at 0 .

INIZIALIZATION delivers $r_{0}^{\max }=3, \mathcal{J}_{0}=0$, and

$$
\begin{aligned}
& A_{0}=-\underbrace{\left(\begin{array}{c}
-1 \\
0 \\
0
\end{array}\right)}_{\xi_{0}} \underbrace{\left(\begin{array}{ccc}
1 & 0 & 0
\end{array}\right)}_{\eta_{0}^{\prime}}, \quad a_{1}=\alpha_{0}=\xi_{0}, \quad b_{1}=\beta_{0}=\eta_{0}, \\
& \theta_{0}=\bar{\beta}_{0} \bar{\alpha}_{0}^{\prime}=\left(\begin{array}{ccc}
-1 & 0 & 0 \\
0 & 0 & 0 \\
0 & 0 & 0
\end{array}\right) .
\end{aligned}
$$

Given that $1=r_{0}<r_{0}^{\max }=3$, the counter $j$ is increased to $j=1$ and RECURSION 1 delivers $r_{1}^{\max }=2$,

$$
\begin{aligned}
& A_{1,1}=A_{1}=\left(\begin{array}{ccc}
0 & 0 & 0 \\
0 & -1 & 0 \\
1 & 0 & 0
\end{array}\right), \quad D_{1,1}=\theta_{0} A_{1,1}=0, \\
& a_{1 \perp}^{\prime} A_{1,1} b_{1 \perp}=-\underbrace{\left(\begin{array}{l}
1 \\
0
\end{array}\right)}_{\xi_{1}} \underbrace{\left(\begin{array}{cc}
1 & 0
\end{array}\right)}_{\eta_{1}^{\prime}}, \quad \mathcal{J}_{1}=(1,0), \\
& \alpha_{1}=\bar{a}_{1 \perp} \xi_{1}=\left(\begin{array}{l}
0 \\
1 \\
0
\end{array}\right), \quad \beta_{1}=\bar{b}_{1 \perp} \eta_{1}=\left(\begin{array}{l}
0 \\
1 \\
0
\end{array}\right), \quad \theta_{1}=\bar{\beta}_{1} \bar{\alpha}_{1}^{\prime}=\left(\begin{array}{lll}
0 & 0 & 0 \\
0 & 1 & 0 \\
0 & 0 & 0
\end{array}\right),
\end{aligned}
$$

and $a_{2}=\left(\alpha_{1}, \alpha_{0}\right), b_{2}=\left(\beta_{1}, \beta_{0}\right), a_{2 \perp}=b_{2 \perp}=(0,0,1)^{\prime}$. Since $1=r_{1}<r_{1}^{\max }=2$, the counter $j$ is incremented to $j=2$ and RECURSION 2 delivers $r_{2}^{\max }=1$,

$$
A_{1,2}=A_{2}=\left(\begin{array}{ccc}
0 & 0 & -\frac{1}{2} \\
0 & 0 & 0 \\
\frac{1}{2} & 0 & 0
\end{array}\right), \quad D_{1,2}=\theta_{0} A_{1,2}=\left(\begin{array}{ccc}
0 & 0 & \frac{1}{2} \\
0 & 0 & 0 \\
0 & 0 & 0
\end{array}\right),
$$

and because $D_{1,1}=0$, 


$$
A_{2,1}=A_{1,2}=\left(\begin{array}{ccc}
0 & 0 & -\frac{1}{2} \\
0 & 0 & 0 \\
\frac{1}{2} & 0 & 0
\end{array}\right), \quad D_{2,1}=\theta_{1} A_{2,1}=0
$$

Next, one finds that $a_{2 \perp}^{\prime} A_{2,1} b_{2 \perp}=0$ and hence $\mathcal{J}_{2}=\mathcal{J}_{1}, a_{3}=a_{2}, b_{3}=b_{2}$, and $\theta_{2}=0$. Because $0=r_{2}<r_{2}^{\max }=1$, the counter is upgraded to $j=3$ and RECURSION 3 delivers $r_{3}^{\max }=1$,

$$
\begin{gathered}
A_{1,3}=A_{3}=\left(\begin{array}{ccc}
0 & 0 & -\frac{1}{2} \\
0 & 0 & 0 \\
0 & 0 & -\frac{1}{4}
\end{array}\right), \quad D_{1,3}=\theta_{0} A_{1,3}=\left(\begin{array}{ccc}
0 & 0 & \frac{1}{2} \\
0 & 0 & 0 \\
0 & 0 & 0
\end{array}\right), \\
A_{2,2}=A_{1,3}+A_{1,1} D_{1,2}=\left(\begin{array}{ccc}
0 & 0 & -\frac{1}{2} \\
0 & 0 & 0 \\
0 & 0 & 0
\end{array}\right), D_{2,2}=D_{1,2}+\theta_{1} A_{2,2}=\left(\begin{array}{ccc}
0 & 0 & \frac{1}{2} \\
0 & 0 & 0 \\
0 & 0 & 0
\end{array}\right),
\end{gathered}
$$

and

$$
A_{3,1}=A_{2,2}+A_{2,1} D_{2,1}=\left(\begin{array}{ccc}
0 & 0 & -\frac{1}{2} \\
0 & 0 & 0 \\
0 & 0 & 0
\end{array}\right), \quad D_{3,1}=D_{2,1}+\theta_{2} A_{3,1}=0 .
$$

Hence one finds that $a_{3 \perp}^{\prime} A_{3,1} b_{3 \perp}=0$ so that $\mathcal{J}_{3}=\mathcal{J}_{2}, a_{4}=a_{3}, b_{4}=b_{3}$, and $\theta_{3}=0$. Because $0=r_{3}<r_{3}^{\max }=1$, the counter $j$ is raised to $j=4$ and RECURSION 4 delivers $r_{4}^{\max }=1, A_{1,4}=A_{4}:=0, D_{1,4}=\theta_{0} A_{1,4}=0$,

$$
\begin{gathered}
A_{2,3}=A_{1,4}+A_{1,1} D_{1,3}=\left(\begin{array}{ccc}
0 & 0 & 0 \\
0 & 0 & 0 \\
0 & 0 & \frac{1}{4}
\end{array}\right), \quad D_{2,3}=D_{1,3}+\theta_{1} A_{2,3}=\left(\begin{array}{ccc}
0 & 0 & \frac{1}{2} \\
0 & 0 & 0 \\
0 & 0 & 0
\end{array}\right), \\
A_{3,2}=A_{2,3}+A_{2,1} D_{2,2}=\left(\begin{array}{ccc}
0 & 0 & 0 \\
0 & 0 & 0 \\
0 & 0 & \frac{1}{2}
\end{array}\right), \quad D_{3,2}=D_{2,2}+\theta_{2} A_{3,2}=\left(\begin{array}{ccc}
0 & 0 & \frac{1}{2} \\
0 & 0 & 0 \\
0 & 0 & 0
\end{array}\right), \\
A_{4,1}=A_{3,2}+A_{3,1} D_{3,1}=\left(\begin{array}{ccc}
0 & 0 & 0 \\
0 & 0 & 0 \\
0 & 0 & \frac{1}{2}
\end{array}\right), \quad D_{4,1}=D_{3,1}+\theta_{3} A_{4,1}=0 .
\end{gathered}
$$

Hence one has $a_{4 \perp}^{\prime} A_{4,1} b_{4 \perp}=\frac{1}{2}, \xi_{4}=1, \eta_{4}=-\frac{1}{2}, \mathcal{J}_{4}=(4,1,0)$, and

$$
\alpha_{4}=\bar{a}_{4 \perp} \xi_{4}=\left(\begin{array}{l}
0 \\
0 \\
1
\end{array}\right), \quad \beta_{4}=\bar{b}_{4 \perp} \eta_{4}=\left(\begin{array}{c}
0 \\
0 \\
-\frac{1}{2}
\end{array}\right), \quad \theta_{4}=\bar{\beta}_{4} \bar{\alpha}_{4}^{\prime}=\left(\begin{array}{ccc}
0 & 0 & 0 \\
0 & 0 & 0 \\
0 & 0 & -2
\end{array}\right) \text {, }
$$

$a_{5}=\left(\alpha_{4}, \alpha_{1}, \alpha_{0}\right), b_{5}=\left(\beta_{4}, \beta_{1}, \beta_{0}\right)$.

Given that the full rank condition $1=r_{4}=r_{4}^{\max }=1$ is reached, the algorithm enters the FinAL LOOPS and defines $m=j=4, \mathcal{J}=\mathcal{J}_{4}=(4,1,0), a=a_{5}, b=b_{5}$. Setting $q=m=4$, one can then compute $D_{k}=D_{5, k}$ for $k=1,2,3$ and $C_{k}=E_{5, k}$ for $k=0,1,2,3$ using (5.2) and (5.4); one finds that

$$
\begin{gathered}
D_{1}=\left(\begin{array}{ccc}
0 & 0 & 0 \\
0 & 0 & 0 \\
0 & 0 & -\frac{1}{2}
\end{array}\right), \quad D_{2}=D_{3}=\left(\begin{array}{ccc}
0 & 0 & \frac{1}{2} \\
0 & 0 & 0 \\
0 & 0 & 0
\end{array}\right), \\
C_{0}=\left(\begin{array}{lll}
0 & 0 & 0 \\
0 & 0 & 0 \\
0 & 0 & 2
\end{array}\right), \quad C_{1}=C_{2}=\left(\begin{array}{ccc}
0 & 0 & 0 \\
0 & 0 & 0 \\
-1 & 0 & 0
\end{array}\right), \quad C_{3}=\left(\begin{array}{ccc}
0 & 0 & 0 \\
0 & -1 & 0 \\
0 & 0 & 0
\end{array}\right) .
\end{gathered}
$$


Hence (see Theorem 3.5), $A(z)$ has a pole of order $m=4$ at $z_{0}=0$ and the coefficients of the principal part of $A(z)^{-1}$ at $z_{0}=0$ are given by

$$
\begin{aligned}
& B_{0}=C_{0}=\left(\begin{array}{lll}
0 & 0 & 0 \\
0 & 0 & 0 \\
0 & 0 & 2
\end{array}\right), \quad B_{1}=C_{1}+D_{1} B_{0}=\left(\begin{array}{ccc}
0 & 0 & 0 \\
0 & 0 & 0 \\
-1 & 0 & -1
\end{array}\right), \\
& B_{2}=C_{2}+D_{1} B_{1}+D_{2} B_{0}=\left(\begin{array}{ccc}
0 & 0 & 1 \\
0 & 0 & 0 \\
-\frac{1}{2} & 0 & \frac{1}{2}
\end{array}\right), \\
& B_{3}=C_{3}+D_{1} B_{2}+D_{2} B_{1}+D_{3} B_{0}=\left(\begin{array}{ccc}
-\frac{1}{2} & 0 & \frac{1}{2} \\
0 & -1 & 0 \\
\frac{1}{4} & 0 & -\frac{1}{4}
\end{array}\right) .
\end{aligned}
$$

A direct computation shows that (1.3) is satisfied. Because $m=4, p=3$, and $p-r_{0}=2$, one has $m>\frac{p^{3}}{\left(p-r_{0}\right)^{3}}$, and thus a computational gain arises by performing the complete reduction process instead of the one-step reduction.

Finally, construct the local Smith form and a Jordan pair of $A(z)$ at 0 using Theorems 4.2 and 4.3 and Corollary 4.4. From $\mathcal{J}=(4,1,0)$ and $r_{4}=r_{1}=r_{0}=1$ one has $\mathcal{K}=\mathcal{J}_{+}=(4,1)$; see Definition 4.1.

From (4.2) the local Smith form of $A(z)$ at $z_{0}=0$ is

$$
\Lambda(z):=\operatorname{diag}\left(\left(z-z_{0}\right)^{j} I_{r_{j}}\right)_{j \in \mathcal{J}}=\left(\begin{array}{ccc}
z^{4} & 0 & 0 \\
0 & z & 0 \\
0 & 0 & 1
\end{array}\right) .
$$

From Corollary 4.4 one has that a Jordan pair $(X, J)$ of $A(z)$ at $z_{0}=0$ is

$$
X:=\left(X_{i}\right)_{i=1}^{p-r_{0}}=\left(X_{1}, X_{2}\right), \quad J:=\operatorname{diag}\left(I_{r_{j}} \otimes J_{j}\right)_{j \in \mathcal{J}_{+}}=\left(\begin{array}{cc}
J_{4} & 0 \\
0 & J_{1}
\end{array}\right),
$$

where

$$
\begin{array}{ll}
X_{1}:=\left(\phi_{1, n}\right)_{n=0}^{k_{1}-1}=\left(\phi_{1,0}, \phi_{1,1}, \phi_{1,2}, \phi_{1,3}\right), \quad J_{4}=\left(\begin{array}{cccc}
0 & 1 & & \\
& 0 & 1 & \\
& & 0 & 1 \\
& & 0
\end{array}\right), \\
X_{2}:=\left(\phi_{2, n}\right)_{n=0}^{k_{2}-1}=\phi_{2,0}, \quad J_{1}=0,
\end{array}
$$

and $\phi_{i, n}$ is the $i$ th column of $\Phi_{n}$. Using (4.7), the latter is computed as

$$
\begin{aligned}
& \Phi_{0}=\left(\bar{\beta}_{4}, \bar{\beta}_{1}, \bar{\beta}_{0}\right), \quad \Phi_{1}=D_{3,1} \Phi_{0}, \\
& \Phi_{2}=D_{3,1} \Phi_{1}+D_{2,2} \Phi_{0}, \Phi_{3}=D_{3,1} \Phi_{2}+D_{2,2} \Phi_{1}+D_{1,3} \Phi_{0}
\end{aligned}
$$

and found to be

$$
\Phi_{0}=\left(\begin{array}{ccc}
0 & 0 & 1 \\
0 & 1 & 0 \\
-2 & 0 & 0
\end{array}\right), \quad \Phi_{1}=0, \quad \Phi_{2}=\Phi_{3}=\left(\begin{array}{ccc}
-1 & 0 & 0 \\
0 & 0 & 0 \\
0 & 0 & 0
\end{array}\right)
$$

hence 


$$
X_{1}=\left(\phi_{1,0}, \phi_{1,1}, \phi_{1,2}, \phi_{1,3}\right)=\left(\begin{array}{cccc}
0 & 0 & -1 & -1 \\
0 & 0 & 0 & 0 \\
-2 & 0 & 0 & 0
\end{array}\right), \quad X_{2}=\phi_{2,0}=\left(\begin{array}{l}
0 \\
1 \\
0
\end{array}\right) .
$$

Application of Theorem 7.1 on p. 184 in [15] confirms that $(X, J)$ is a Jordan pair of $A(z)$ at 0 .

8. Conclusion. The ELRF delivers a recursive formula to compute the order of the pole and the Laurent coefficients of the inverse of a regular analytic matrix function, without stacking coefficients in potentially large linear systems. The procedure consists in performing a finite sequence of rank factorizations of matrices of nonincreasing dimension at most equal to the dimension of the original matrix function.

The sequence of rank factorizations is shown to deliver the partial multiplicities and the number of partial multiplicities of a given value, i.e., the local Smith form of the original matrix function, and to provide a construction of an extended canonical system of root functions of $A(z)$ at $z_{0}$. In this way, the structure of Jordan pairs is fully characterized and full information on Jordan chains is available.

Moreover, the CRP in [3] is shown here to coincide with the ELRF; via this equivalence, one has that the number of reductions in the CRP is equal to the number of distinct nonzero partial multiplicities and each reduction step decreases the dimension of the coefficients by the number of partial multiplicities that are equal to a given value. This links the characteristics of the reduction process to the structure of the local Smith form. Finally, it is shown that the computational complexity of the ELRF compares favourably with the one of the one-step reduction process.

\section{REFERENCES}

[1] A. Albrecht, P. Howlett, and C. Pearce, The fundamental equations for inversion of operator pencils on Banach space, J. Math. Anal. Appl., 413 (2014), pp. 411-421, doi:10. 1016/j.jmaa.2013.11.060.

[2] K. E. Avrachenkov, J. A. Filar, and P. G. Howlett, Analytic Perturbation Theory and Its Applications, SIAM, Philadelphia, 2013, doi:10.1137/1.9781611973143.

[3] K. E. Avrachenkov, M. Haviv, and P. G. Howlett, Inversion of analytic matrix functions that are singular at the origin, SIAM J. Matrix Anal. Appl., 22 (2001), pp. 1175-1189, doi: $10.1137 /$ S0895479898337555.

[4] H. Bart, I. Gohberg, M. KaAshoek, And A. Ran, Factorization of Matrix and Operator Functions: The State Space Method, Birkhäuser, Basel, Boston, Berlin, 2008, doi:10.1007/ 978-3-7643-8268-1.

[5] H. Baumgärtel, Analytic Perturbation Theory for Matrices and Operators, Birkhäuser, Basel, Boston, Berlin, 1985.

[6] A. Ben-Israel and T. Greville, Generalized Inverses: Theory and Applications, 2nd ed., Springer, New York, 2003, doi:10.1007/b97366.

[7] R. Engle And C. Granger, Co-integration and error correction: Representation, estimation, and testing, Econometrica, 55 (1987), pp. 251-276, doi:10.2307/1913236.

[8] M. Franchi, A representation theory for polynomial cofractionality in vector autoregressive models, Econometric Theory, 26 (2010), pp. 1201-1217, doi:10.1017/s0266466609990508.

[9] M. Franchi and P. Paruolo, A characterization of vector autoregressive processes with common cyclical features, J. Econometrics, 163 (2011), pp. 105-117, doi:10.1016/j.jeconom. 2010.11.009.

[10] M. Franchi and P. Paruolo, Inversion of regular analytic matrix functions: Local Smith form and subspace duality, Linear Algebra Appl., 435 (2011), pp. 2896-2912, doi:10.1016/ j.laa.2011.05.005.

[11] F. Gantmacher, The Theory of Matrices, Vol. I, AMS Chelsea, Providence, RI, 1959.

[12] I. Gohberg, S. Goldberg, And M. A. KaAshoek, Classes of Linear Operators, Vol. 1, Oper. Theory Adv. Appl. 49, Birkhäuser Verlag, Basel, 1990, doi:10.1007/978-3-0348-7509-7.

[13] I. Gohberg, M. KaAshoek, And F. van Schagen, On the local theory of regular analytic matrix functions, Linear Algebra Appl., 182 (1993), pp. 9-25, doi:10.1016/ 
0024-3795(93)90488-a.

[14] I. Gohberg, M. KaAshoek, and F. van Schagen, Partially Specified Matrices and Operators: Classification, Completion, Applications, Birkhäuser, Basel, Boston, Berlin, 1995, doi:10. 1007/978-3-0348-9100-4.

[15] I. Gohberg, P. Lancaster, And L. Rodman, Matrix Polynomials, Academic Press, New York, 1982; reprinted, SIAM, Philadelphia, 2009, doi:10.1137/1.9780898719024.

[16] I. Gohberg, P. Lancaster, And L. Rodman, Invariant Subspaces of Matrices with Applications, Wiley, New York, 1986; reprinted, SIAM, Philadelphia, 2006, doi:10.1137/1. 9780898719093.

[17] I. Gohberg AND E. Sigal, An operator generalization of the logarithmic residue theorem and the theorem of Rouché, Math. USSR-Sb., 13 (1971), pp. 603-625, doi:10.1070/ sm1971v013n04abeh003702.

[18] G. Golub And C. Van Loan, Matrix Computations, The Johns Hopkins University Press, Baltimore, London, 1996.

[19] N. Haldrup AND M. SAlmon, Representation of I(2) cointegrated systems using the Smith-McMillan form, J. Econometrics, 44 (1998), pp. 303-325, doi:10.1016/ s0304-4076(97)00088-2.

[20] M. Haviv AND Y. Ritov, On series expansions and stochastic matrices, SIAM J. Matrix Anal. Appl., 14 (1993), pp. 670-676, doi:10.1137/0614047.

[21] M. Haviv, Y. Ritov, And U. Rothblum, Taylor expansions of eigenvalues of perturbed matrices with applications to spectral radii of nonnegative matrices, Linear Algebra Appl., 168 (1992), pp. 159-188, doi:10.1016/0024-3795(92)90293-J.

[22] P. Howlett, Input retrieval in finite dimensional linear systems, J. Austral. Math. Soc. Ser. B, 23 (1982), pp. 357-382, doi:10.1017/s033427000000031x.

[23] P. Howlett, K. Avrachenkov, C. Pearce, and V. Ejov, Inversion of analytically perturbed linear operators that are singular at the origin, J. Math. Anal. Appl., 353 (2009), pp. 68-84, doi:10.1016/j.jmaa.2008.11.074.

[24] S. JohANSEN, Estimation and hypothesis testing of cointegration vectors in Gaussian vector autoregressive models, Econometrica, 59 (1991), pp. 1551-1580, doi:10.2307/2938278.

[25] S. Johansen, A representation of vector autoregressive processes integrated of order 2, Econometric Theory, 8 (1992), pp. 188-202, doi:10.1017/s0266466600012755.

[26] S. Johansen, Likelihood-Based Inference in Cointegrated Vector Autoregressive Models, Oxford University Press, New York, 1996, doi:10.1093/0198774508.001.0001.

[27] T. Kailath, Linear Systems, Prentice-Hall, Englewood Cliffs, NJ, 1980.

[28] M. KELDYSH, On eigenvalues and eigenfunctions of certain classes of non self-adjoint operators, Dokl. Akad. Nauk SSSR, 77 (1951), pp. 11-14 (in Russian).

[29] P. Lancaster and M. Tismenetsky, The Theory of Matrices, Academic Press, Orlando, FL, 1985.

[30] C. LANGenhop, The Laurent expansion for a nearly singular matrix, Linear Algebra Appl., 4 (1971), pp. 329-340, doi:10.1016/0024-3795(71)90004-8.

[31] A. Markus, Introduction to the Spectral Theory of Polynomial Operator Pencils, Transl. Math. Monogr. 71, AMS, Providence, RI, 1988.

[32] L. Rodman, An Introduction to Operator Polynomials, Birkhäuser, Basel, Boston, Berlin, 1989, doi:10.1007/978-3-0348-9152-3.

[33] M. SAIn AND J. MASsEY, Invertibility of linear time invariant dynamical systems, IEEE Trans. Automat. Control, AC-14 (1969), pp. 141-149, doi:10.1109/tac.1969.1099133.

[34] P. SChWEITZER AND G. STEWART, The Laurent expansion of pencils that are singular at the origin, Linear Algebra Appl., 183 (1993), pp. 237-254, doi:10.1016/0024-3795(93)90435-q.

[35] F. Vahid and R. Engle, Codependent cycles, J. Econometrics, 80 (1997), pp. 199-221, doi:10. 1016/s0304-4076(97)00032-8.

[36] M. VISHIK AND L. LyUSTERNIK, The solution of some perturbation problems for matrices and selfadjoint or non-selfadjoint differential equations, Uspekhi Mat. Nauk, 15 (1960), pp. 380 (in Russian); Russian Math. Surveys, 15 (1960), pp. 1-73 (in English), doi:10.1070/ rm1960v015n03abeh004092.

[37] J. Wilkening, An algorithm for computing Jordan chains and inverting analytic matrix functions, Linear Algebra Appl., 427 (2007), pp. 6-25, doi:10.1016/j.laa.2007.06.012.

[38] J. Wilkening and J. Yu, A local construction of the Smith normal form of a matrix polynomial, J. Symbolic Comput., 46 (2011), pp. 1-22, doi:10.1016/j.jsc.2010.06.025. 